\title{
Influenza $\mathrm{A} M 2$ recruits $\mathrm{M} 1$ to the plasma membrane: a fluorescence fluctuation microscopy study
}

Annett Petrich ${ }^{1}$, Valentin Dunsing ${ }^{1}$, Sara Bobone ${ }^{2}$, Salvatore Chiantia ${ }^{1^{*}}$

\section{Author information}

${ }^{1}$ University of Potsdam, Institute of Biochemistry and Biology, Karl-Liebknecht-Street 24-25, 14476 Potsdam, Germany

${ }^{2}$ University of Rome Tor Vergata, Department of Chemical Science and Technologies, Via della Ricerca Scientifica 1, 00133 Roma

* Correspondence: chiantia@uni-potsdam.de; Tel.: +49-331-9775872

ORCID IDs:

A.P., 0000-0002-2372-826X

V.D., 0000-0003-2482-1498

S.B., 0000-0001-7975-3803

S.C., 0000-0003-0791-967X

Running title: $M 2$ recruits $M 1$ to the plasma membrane 


\begin{abstract}
Influenza A virus (IAV) is a respiratory pathogen that causes seasonal epidemics with significant mortality. One of the most abundant proteins in IAV particles is the matrix protein 1 (M1), which is essential for the virus structural stability. M1 organizes virion assembly and budding at the plasma membrane (PM), where it interacts with other viral components. The recruitment of $\mathrm{M} 1$ to the $\mathrm{PM}$ as well as its interaction with the other viral envelope proteins (hemagglutinin (HA), neuraminidase, matrix protein $2(\mathrm{M} 2)$ ) is controversially discussed in previous studies. Therefore, we used fluorescence fluctuation microscopy techniques (i.e., scanning fluorescence cross-correlation spectroscopy and Number and Brightness) to quantify the oligomeric state of $\mathrm{M} 1$ and its interactions with other viral proteins in co-transfected as well as infected cells. Our results indicate that $\mathrm{M} 1$ is recruited to the PM by $M 2$, as a consequence of the strong interaction between the two proteins. In contrast, only a weak interaction between M1 and HA was observed. M1-HA interaction occurred only in the case that $\mathrm{M} 1$ was already bound to the PM. We therefore conclude that $\mathrm{M} 2$ initiates the assembly of IAV by recruiting M1 to the PM, possibly allowing its further interaction with other viral proteins.
\end{abstract}

\title{
Statement of Significance
}

Influenza A virus (IAV) is a pathogen responsible for epidemics and occasional pandemics and, therefore, a significant burden on health systems. To develop innovative therapeutic approaches, a deeper understanding of the viral replication cycle is needed. For example, during the formation of new virions in infected cells, several viral components must assemble at the plasma membrane, but the molecular interactions involved in this process are not clearly understood. In this work, we use quantitative fluorescence microscopy methods to monitor the interplay between several viral proteins in live cell models. Our results underline the importance of the interactions between two specific proteins (M1 and M2) and shed light on the first steps in IAV assembly.

\section{Keywords}

fluorescence correlation spectroscopy, fluorescence fluctuation spectroscopy, fluorescence microscopy, hemagglutinin, influenza, matrix protein 1 , matrix protein 2 , neuraminidase, Number and Brightness, plasma membrane, protein-protein interaction, virus assembly

\section{Abbreviations}

ACF, autocorrelation function; AF488, Alexa Fluor ${ }^{\circledR} 488$; CCF, cross-correlation function; (cc)N\&B, (cross-correlation) number and brightness; FP, fluorescence protein; FPV, influenza A/FPV/Rostock/1934 virus mutant 1; HA, hemagglutinin protein; IAV, influenza A virus; M1, IAV matrix protein 1, M2, IAV matrix protein 2; mEGFP, monomeric enhanced green fluorescent protein; $\mathrm{mp}$, myristoylated and palmitoylated; $N A$, neuraminidase protein; $\mathrm{p}_{\mathrm{f}}$, 
bioRxiv preprint doi: https://doi.org/10.1101/2021.05.06.442926; this version posted October 16, 2021. The copyright holder for this preprint (which was not certified by peer review) is the author/funder, who has granted bioRxiv a license to display the preprint in perpetuity. It is made available under aCC-BY-NC-ND 4.0 International license.

fluorescence probability; PM, plasma membrane; rel. cc., relative cross-correlation; sFCCS, scanning fluorescence cross-correlation spectroscopy; vRNPs, viral ribonucleoproteins 


\section{Introduction}

Influenza A viruses (IAVs) belong to the family of the Orthomyxoviridae. These pathogens represent a substantial global health burden, being associated with significant morbidity and mortality through frequent epidemics and several pandemics $(1,2)$. IAV is enveloped by a lipid bilayer that is derived from the host cell membrane and contains two integral transmembrane glycoproteins (i.e. hemagglutinin (HA) and neuraminidase (NA)) and one transmembrane protein with a proton-selective ion channel activity (i.e. the matrix protein $2(M 2))(3,4)$. The envelope protein HA is a homotrimeric type I transmembrane glycoprotein and is the major surface protein of IAV particles (5-7). HA plays a major role in viral entry by mediating the attachment of the virus to cell surface sialic acid molecules, membrane fusion after internalization, and the release of viral genome into target cells (5-8). The surface protein NA is a homotetrameric type II transmembrane glycoprotein that facilitates the release of newly synthesized virus particles from the infected cells by enzymatic cleavage of the cell surface receptor molecules (5-8). Additionally, a small amount of homotetrameric M2 molecules are embedded in the viral envelope (approximately 16 to 20 molecules in a virus, compared to ca. 300-400 HA and 50 NA copies) $(6,7) . M 2$ is a type III transmembrane protein which functions as proton channel activated by acidic $\mathrm{pH}$ and is important for genome unpacking during virus entry (7-9). Moreover, it was shown that M2 is connected to virus morphology, production of infectious virus particles, and membrane scission (9-13). All the three envelope proteins are transported from the trans-Golgi network to the apical plasma membrane via the secretory pathway $(8,9,14)$. Both glycoproteins, HA and NA, are supposed to be enriched in lipid "raft" microdomains at the virion budding site, whereas $M 2$ was suggested to localize to the edges of such domains $(8,14-16)$.

The luminal side of the viral envelope is coated with the matrix protein 1 (M1), which forms the viral nucleocapsid in close contact to the lipid membrane (17-20), binds the viral ribonucleoproteins (VRNPs) $(4,21)$, and is supposed to interact with viral surface proteins (10, 11, 22-24). Moreover, M1 is the most abundant, highly conserved protein in IAV particles and is important for several processes during viral replication, including the regulation of capsid disassembly, virus budding and morphogenesis $(3,8,25)$. Interestingly, M1 lacks an apical transport signal, implying that the membrane localization of $\mathrm{M} 1$ in infected cells might be due to piggyback transport with HA, NA, M2 or vRNPs $(26,27)$. For this reason, various hypotheses 
regarding the association of $\mathrm{M} 1$ to the plasma membrane (PM) have been proposed over the years. First, several studies established that M1 associates with negatively charged lipids in model membranes $(17-20,28,29)$. Nevertheless, such interactions appear not to be sufficient for the actual association of M1 to the PM in non-infected cells (i.e. in cells expressing M1 as the only viral protein) $(17,27)$. Accordingly, M1 was proposed to interact with the cytoplasmic tails of HA and NA during their apical transport (22-24, 30,31), as well as with the cytoplasmic tails of $M 2$ at the assembly site $(10,11,27)$. Interactions between $M 1$ and $H A, N A$, or M2 have been investigated via bulk biochemistry methods (e.g. by altered detergent solubility $(22,24)$, increased membrane association (31) of $M 1$ in the presence of HA or NA, or coimmunoprecipitation of $M 1$ in the presence of $M 2(10,11,32))$. Nevertheless, no clear consensus has been reached regarding the role of $H A, N A$ or $M 2$ in recruiting M1 to the PM and its subsequent incorporation into virions $(11,33-37)$. In conclusion, the molecular mechanisms involved in M1-driven IAV assembly are not fully understood and the specific interactions between $\mathrm{M} 1$ and other viral surface proteins have not yet been quantified directly in living cells.

To obtain quantitative information on how protein-protein interactions (e.g. M1-M1 or M1$\mathrm{HA}$ ) occur in the native cellular environment, minimally invasive approaches (e.g. fluorescence fluctuation spectroscopy) are needed (38). Here, we apply (cross-correlation) Number and Brightness ( $N \& B$ and $c c N \& B$ ) as well as scanning fluorescence (cross-)correlation spectroscopy (sFCS and sFCCS) analysis in living cells to quantify oligomeric state, concentration and diffusion dynamics of the viral envelope proteins (HA, NA, M2) and $M 1$, as well as their interactions. Our results suggest the presence of a strong interaction between $M 1$ and $M 2$, leading to the recruitment of $\mathrm{M} 1$ to the $\mathrm{PM}$ in a $\mathrm{M} 2$ concentration-dependent manner. We further hypothesize that the interaction between M1 and HA occurs in a subsequent step. Finally, we provide the first experimental evidence of a possible M2 binding-site within the Nterminal domain of M1. 


\section{Materials and Methods}

\section{Plasmids and cloning.}

The plasmids for the transcription and translation of influenza virus RNAs and proteins of the influenza A/FPV/Rostock/1934 virus (H7N1; FPV) mutant 1 were obtained from Michael Veit (Free University, Berlin, Germany), and previously described $(39,40)$. The plasmids encoding the fluorescence proteins (FP) mEGFP or mCherry2 linked to a myristoylated and palmitoylated peptide (mp-mEGFP, mp-mCherry2, mp-2x-mEGFP), and the plasmids for cytosolic expression of mEGFP, 2x-mEGFP were previously described (41) and are available on Addgene (Watertown, MA, USA). The plasmids encoding the FP hetero-dimer mCherry2mEGFP linked to a myristoylated and palmitoylated peptide (mp-mCherry2-mEGFP), and the matrix protein $2(\mathrm{M} 2)$ of FPV with mCherry2 fused to the extracellular terminus of $\mathrm{M} 2$ (mCherry2-M2) were previously described (42). Further information regarding other plasmids and constructs used in this work is provided in the Supplemental Information (SI).

\section{Cell culture and virus infection}

Human embryonic kidney (HEK) cells from the 293T line (CRL-3216TM, purchased from ATCC ${ }^{\circledR}$, Kielpin Lomianki, Poland), and Madin-Darby canine kidney type II (MDCK II) cells (ECACC 00062107, European Collection of Authenticated Cell Cultures, Porton Down, UK) were cultured in phenol red-free Dulbecco's modified Eagle medium (DMEM) with $10 \%$ fetal bovine serum, $2 \mathrm{mM} \mathrm{L-glutamine,} 100 \mathrm{U} / \mathrm{mL}$ penicillin, and $100 \mu \mathrm{g} / \mathrm{mL}$ streptomycin at $37^{\circ} \mathrm{C}$ and $5 \%$ $\mathrm{CO}_{2}$. Cells were passaged every 2-3 days when they reached nearly $80 \%$ confluence in tissue culture flask, for no more than 15 times. All solutions, buffers, and media used for cell culture were purchased from PAN-Biotech (Aidenbach, Germany).

For immunostaining experiments, dishes were coated with a $0.01 \%(\mathrm{w} / \mathrm{v})$ poly-L-lysine solution (MW 150,000 - 300,000 Da, Sigma-Aldrich, Munich, Germany) before cell seeding.

Information regarding virus propagation, titration and infection is provided in the SI. 


\section{Immunofluorescence.}

Transfected and infected cells were fixed at the indicated time points with $4 \%(\mathrm{w} / \mathrm{v})$ paraformaldeyde (Sigma Aldrich, Taufkirchen, Germany) in DPBS+/+. After 15 min, cells were washed three times with DPBS+/+. Permeabilization was performed with $0.1 \%$ (v/v) Triton X$100^{\circledR}$ (Sigma Aldrich, Taufkirchen, Germany) for $10 \mathrm{~min}$, and subsequently washed three times with DPBS+/+. Afterwards, cells were incubated with $2 \%(w / v)$ BSA (Sigma Aldrich, Taufkirchen, Germany) in DPBS+/+ for one hour at room temperature. Primary antibody (monoclonal mouse anti-influenza A M2, clone 14C2 (\#ab5416, abcam, Cambridge, UK), monoclonal mouse anti-influenza A H7 (\#3HI7, HyTest Ltd, Turku, Finland), Clone monoclonal mouse anti-influenza A N1, clone \#2F10E12G1 (\#AB_2860298, SinoBiological, Eschborn, Germany), monoclonal mouse anti-influenza nucleoprotein, clone A1 (\#MAB8257, Millipore trademark of Merck KGaA, Darmstadt,Germany)) were diluted 1:200 or 1:1000 in $0.2 \%(\mathrm{w} / \mathrm{v})$ BSA in DPBS+/+, and incubated overnight at $4{ }^{\circ} \mathrm{C}$. After three washing steps with DPBS+/+, cells were incubated with the 1:1000 diluted secondary antibodies (goat anti-mouse AlexaFluor $^{\circledR}$ 488-conjugated or AlexaFluor ${ }^{\circledR}$ 568-conjugated; Thermo Fisher Scientific, Waltham, MA, USA) for one hour at room temperature. Cells were subsequently washed three times with DPBS+/+.

\section{Confocal microscopy imaging.}

Microscopy measurements were performed on a Zeiss LSM780 system (Carl Zeiss, Oberkochen, Germany) using a Plan-Apochromat 40×/1.2 Korr DIC M27 water immersion objective and a 32-channel GaAsP detector array. To decrease out-of-focus light, a pinhole with size corresponding to one airy unit ( $39 \mu \mathrm{m})$ was used. Samples were excited with a 488$\mathrm{nm}$ argon laser and a 561-nm diode laser. Fluorescence was detected between 499 and 552 $\mathrm{nm}$ (mEGFP, AlexaFluor ${ }^{\circledR} 488$ ) and between 570 and $695 \mathrm{~nm}$ (mCherry2), after passing through a MBS 488/561-nm dichroic mirror. For multicolor measurements, fluorophores were excited and detected sequentially for different regions of the spectrum. Confocal imaging was performed with a frame size of $512 \times 512$ pixels.

Further information regarding setup calibration, sFCCS, (cc)N\&B, bi-directional plasmids and multimerization analysis is provided in the SI. A schematic overview of the SFCCS and ccN\&B analysis is shown in Figure S1. 


\section{Statistical analysis.}

Data from at least three independent experiments were pooled and visualized by using GraphPad Prism vs. 9.0.0 (GraphPad Software, LCC, San Diego, CA, USA) or R (R Foundation for Statistical Computing, Vienna, Austria) packages ggplot2 (43), ggpubr (44), and cowplot (45). If not otherwise indicated, data were displayed as box plots with single data points corresponding to measurements in single cells. Median values and whiskers ranging from minimum to maximum values are displayed. Quantities in the main text are given as median \pm IQR. Sample sizes and $p$-values are given in each graph and figure captions, respectively. Statistical significance was tested by using D`Agostino-Pearson normality test followed by the one-way ANOVA analysis and the Bonferroni's multiple comparisons test.

\section{Code availability.}

MATLAB custom-written code is available from the corresponding author upon reasonable request.

\section{Data availability.}

The datasets analyzed during the current study are available from the corresponding author upon reasonable request. 


\section{Results}

\section{$\mathrm{M} 1$ is recruited to the PM by $\mathrm{M} 2$ but not by HA or NA}

Previous studies have shown that the intracellular localization of the Influenza A matrix protein M1 varies between transfected and infected cells $(15,27)$. As a starting point for our investigations, we have therefore characterized the behavior of a M1-mEGFP fluorescent construct derived from the avian IAV strain FPV directly in living HEK293T cells. Protein localization was monitored via confocal microscopy either i) when expressed by itself, ii) in the presence of all other viral proteins (i.e., via the reverse genetic plasmid system and unlabeled M2 termed here as "all"), or iii) in FPV infected cells (Figure $1 \mathrm{~A}, \mathrm{~B}$ ).

Expression of M1-mEGFP alone indicated a homogenous distribution of M1 through the cytosol and the nucleus (Figure $1 \mathrm{~A}$ ), whereas mEGFP-M1 (i.e., mEGFP fused at the N-terminus) formed large, bright aggregates in the cytosolic region in close proximity to the nucleus (data not shown). The localization of M1-mEGFP was similar to what was previously described for unlabeled M1 $(46,47)$. Therefore, this construct was used for all further experiments. Upon co-transfection of all other IAV (unlabeled) proteins, a distinct enrichment of M1-mEGFP at the PM was detectable, with the protein being homogeneously distributed (Figure $1 \mathrm{~A}$ ). A statistical analysis of the frequency of such an occurrence is not trivial since the number of cells effectively transfected with all 9 plasmids is unknown. Nevertheless, a control experiment suggests that most of the successfully transfected cells express several fluorescently tagged proteins at the same time (Figure S2). Also, the probability that a cell expressing M1-mEGFP does not express any other viral protein is estimated to be very low (i.e., < 1\% for a six-plasmid system approximation, Figure S2 B). Therefore, we conclude that the observed enrichment of M1 at the PM is probably due to the presence of at least one other viral protein.

Notably, we observed filamentous structures originating from the PM (Figure $1 \mathrm{~B}$, left, Figure S3 A) that were not present when M1 was substituted by the membrane-anchored mp-mEGFP (Figure S3 A). Cells infected with FPV showed heterogeneous M1 binding to the PM and 
formation of clusters in almost every cell (i.e. > ca. $90 \%$ ) at 24 hpi (Figure 1 A), as previously observed also for unlabeled M1 $(15,16)$. M1-enriched structures at the PM resembling ruffles were even more evident, compared to the case of the reverse genetic plasmid system (Figure 1 B, right, Figure S3 A). The effectiveness of IAV infection was confirmed via immunofluorescence detection of expressed nucleoprotein (ca. $90 \%$ of infected cells, data not shown).

In order to determine whether M1 localization is determined by the presence of other viral proteins at the PM as previously suggested $(15,16)$, M1-mEGFP was co-expressed with either mCherry2-M2, mCherry2-HATMD, or NA-mCherry2 (Figure $1 \mathrm{C}-\mathrm{E}$ ). It is worth noting that these viral proteins are labeled at the extracellular side (so to preserve possible interactions with intracellular M1) and strongly localize at the PM, similarly to their non-fluorescent counterparts $(48,49)$. Fluorescence microscopy imaging indicated the absence of M1-mEGFP localization at the PM in cells co-expressing this protein with mCherry2-HATMD, NA-mCherry2 constructs (Figure 1D-E) or unlabeled HA or NA (Figure S4 A). On the other hand, upon coexpression of M1-mEGFP with mCherry2-M2, clear colocalization of both proteins at the PM was observed (Figure 1C). Unequivocable association of M1-mEGFP to the PM was observed in circa one quarter of the examined cells and appeared qualitatively correlated with the amount of mCherry2-M2 at the PM (Figure S4 B and C). A quantitative analysis of the correlation between the concentrations of the two proteins at the PM is presented in the following paragraphs. 


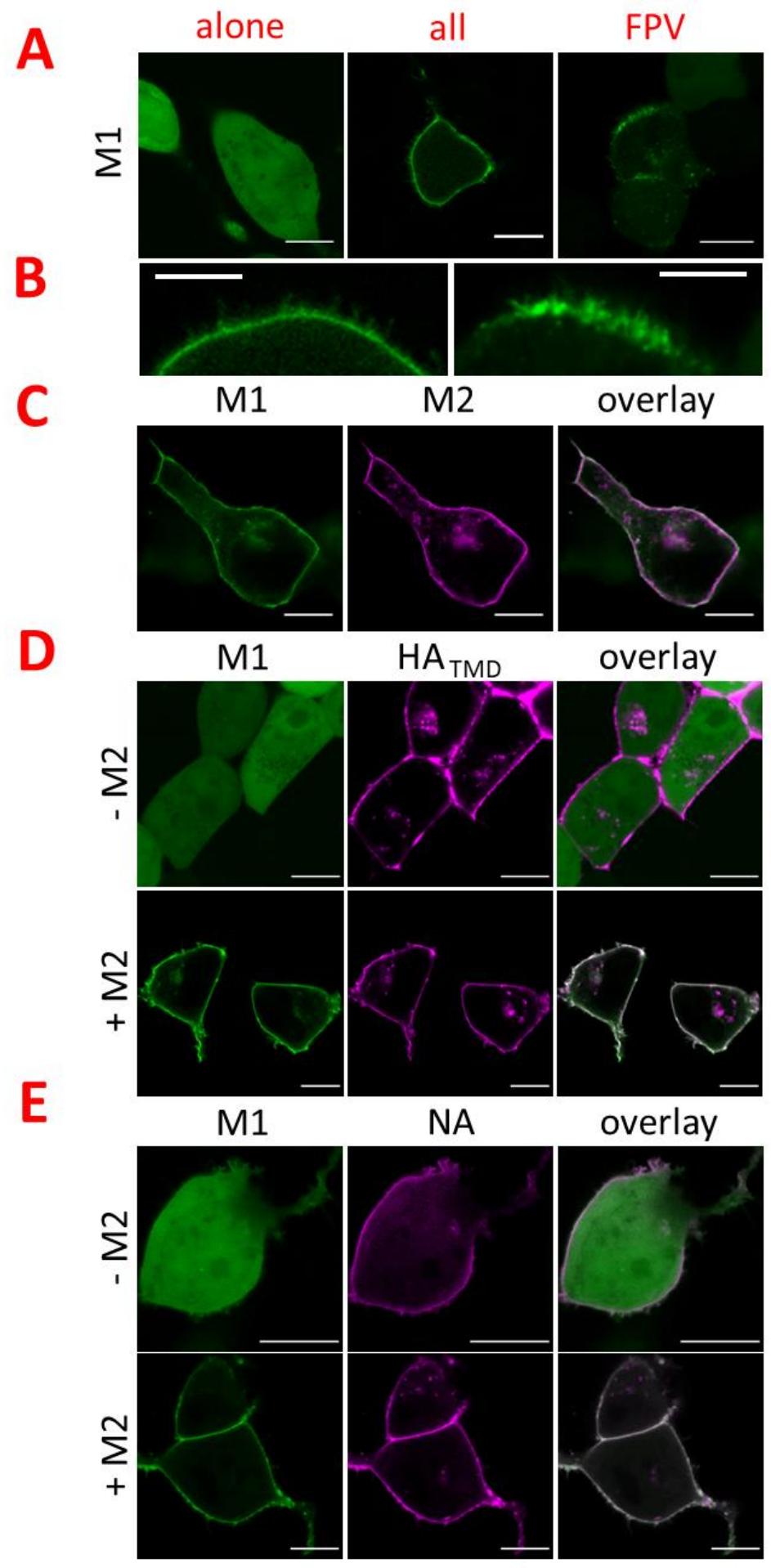

Figure 1: Membrane recruitment of IAV matrix protein 1 (M1) in co-transfected and infected cells. A-B: Representative confocal fluorescence images of HEK293T cells expressing M1-mEGFP (green) from the influenza A/FPV/Rostock/1934 strain (FPV) alone (A, left panel). The same construct was also observed in cells co-transfected with the reverse genetic plasmid system of FPV and unlabeled M2, here called as "all" (A, middle; B, left) and in cells infected with FPV (A, right; B, left). C: Representative confocal fluorescence images of HEK293T cells co-expressing M1-mEGFP (green) and the FPV matrix protein 2 (mCherry2-M2, magenta). The right panels show the two channels merged in a single image. D: Representative confocal fluorescence images of HEK293T cells co-expressing M1-mEGFP (green) and the hemagglutinin (mCherry2-HA $\mathrm{ATD}_{\text {, }}$ magenta) in the absence (upper panels) or in the presence (lower panels) of unlabeled M2. E: Representative confocal fluorescence images of HEK293T cells co-expressing M1-mEGFP (green) and the neuraminidase (NA-mCherry2, magenta) in the absence (upper panels) or in the presence (lower panels) of unlabeled M2. Scale bars represent $10 \mu \mathrm{m}$. 
The membrane distribution of M1-mEGFP was macroscopically homogeneous and no filamentous structures or clustering of M1-mEGFP at the PM were detectable. M2-induced binding of M1-mEGFP to the PM was qualitatively not further influenced by co-expression of mCherry2-HATMD or NA-mCherry2 (Figure 1 D-E).

In conclusion, $\mathrm{M} 2$ seems to be necessary for the recruitment of $\mathrm{M} 1$ to the PM. Also, the lateral organization of this protein on the lipid membrane is influenced by the presence of other viral proteins, as observed in infected cells.

\section{M1 multimeric state at the PM ranges from dimers to large multimers}

In order to quantify the concentration-dependent oligomerization of $M 1, N \& B$ analysis was carried out in infected as well as co-transfected cells (Figure 2). This approach was applied in the past to quantify protein multimerization as a function of local concentration and cellular localization $(50,51)$. Compared to other methods based on fluorescence fluctuation analysis, N\&B provides more representative results in samples characterized by spatial inhomogeneities and slow dynamics (52). The amount of fluorescence signal detected for a single independent protein complex (e.g., a protein dimer) in the unit of time is indicated by the molecular brightness. This parameter is directly connected to the number of fluorophores within such a complex and, therefore, to the multimeric state of the fusion-labeled protein. Specifically, the multimerization can be quantified by normalization of the measured brightness values with the molecular brightness of a monomeric and dimeric reference (see Materials and Methods) (41). To avoid possible interactions between the ectodomain of viral proteins and the solid substrate, we performed all measurements at the equatorial plane of cells rather than the basal membrane (which is often analyzed in the context of N\&B studies). Our data show that protein oligomerization can be reproducibly quantified for both PM regions, without substantial differences (Figure S5).

The fluorescent construct M1-mEGFP described in the previous paragraph was expressed in HEK293T cells either i) in the presence of unlabeled M2, ii) concurrently with the reverse genetic plasmid system and unlabeled M2, named hereafter "all", iii) concurrently with FPV infection or iv) alone (Figure 2). 
The results shown in Figure $2 \mathrm{~A}$ and $\mathrm{B}$ indicate that, upon co-expression of M2, M1-mEGFP does not form large complexes, compared to the case in which other viral proteins are present (i.e., in the case of the reverse genetic plasmid system or of infection). In the latter cases, higher intensity and brightness values are in fact observed at the PM. The average intensity and molecular brightness values were calculated at each pixel of ROls (including e.g., the PM or cytosolic regions) and represented as two dimensional histograms (Figure S6, representative example of data from Figure $2 \mathrm{~A}$ and $\mathrm{B}$ ). The brightness values of M1-mEGFP within each cell were usually symmetrically distributed around their average values for cotransfected cells expressing unlabeled M2, but slightly skewed towards large values in infected cells or cells transfected with the plasmid set "all". The brightness values of such distributions were then normalized using the corresponding monomer and dimer controls (Figure $2 \mathrm{C}$ ). The analysis of cells expressing only M1 indicated that M1-mEGFP in the cytosol has a normalized brightness between 1 and 2 (1.2 \pm 0.7 , median \pm IQR, $n=48$ cells). For comparison, the oligomerization state of cytosolic control monomers (mEGFP) and dimers (mEGFP-mEGFP) is also shown. It is worth noting that $\mathrm{N} \& \mathrm{~B}$ analysis provides an average oligomerization value in the case of mixtures of different multimeric species (51). Therefore, the measured normalized brightness for cytosolic M1-mEGFP suggests that the protein is present as a mixture of e.g. monomers (ca. $80 \%$, assuming $p_{f}=0.7$ ) and dimers (ca. $20 \%$ ) at the observed concentrations. M1-mEGFP oligomerization slightly increased upon binding to the PM in the presence of unlabeled M2 (2.2 \pm 0.6 , median \pm IQR, $n=53$ cells). M1-mEGFP oligomeric state increased significantly upon co-transfection with all other viral proteins ("all", $5.0 \pm 1.6$, median \pm IQR, $n$ = 39 cells), or upon infection (7.2 \pm 5.2 , median $\pm I Q R, n=46$ cells). For comparison, the oligomeric state of control monomers (mp-mEGFP) and dimers (mp-mEGFP-mEGFP) is also shown. Additionally, M1-mEGFP showed a significant concentration-dependent oligomerization behavior in concurrently infected cells and in transfected cells expressing all other viral proteins (Figure $2 \mathrm{D}$ ). On the other hand, the oligomerization of M1-mEGFP in cotransfected cells expressing unlabeled M2 seemed to be independent from concentration and stable around values corresponding, in average, to M1-mEGFP dimers. As also evident from Figure 2 D, higher concentrations of M1-mEGFP at the PM were observed in general in infected cells, as well as in co-transfected cells expressing all other viral proteins. 
A

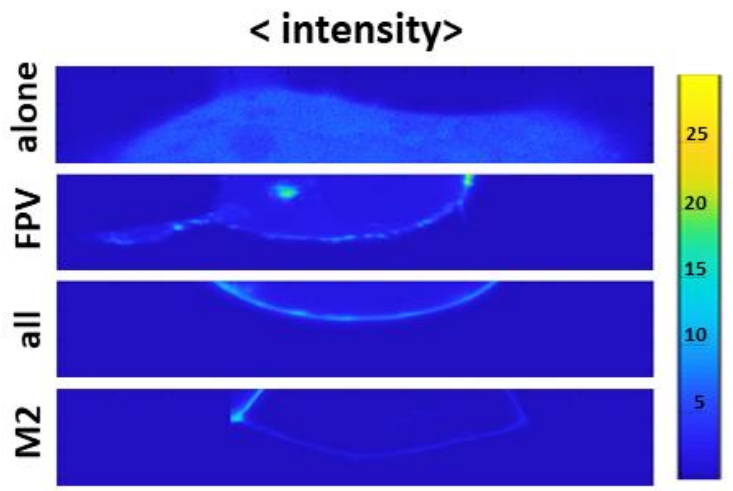

B
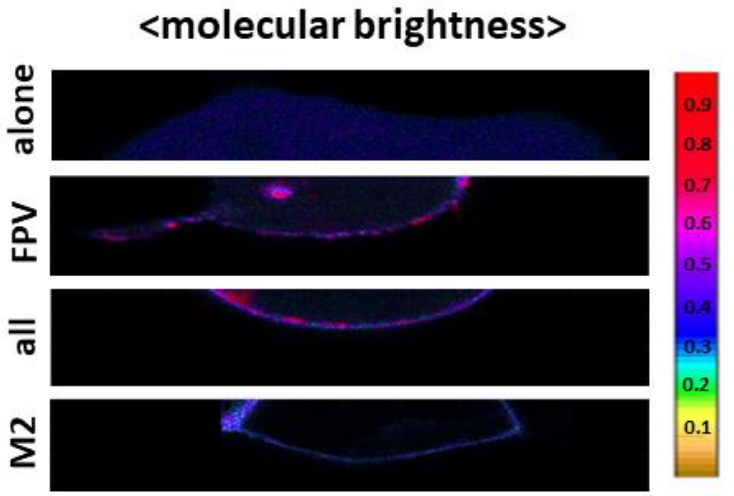

D

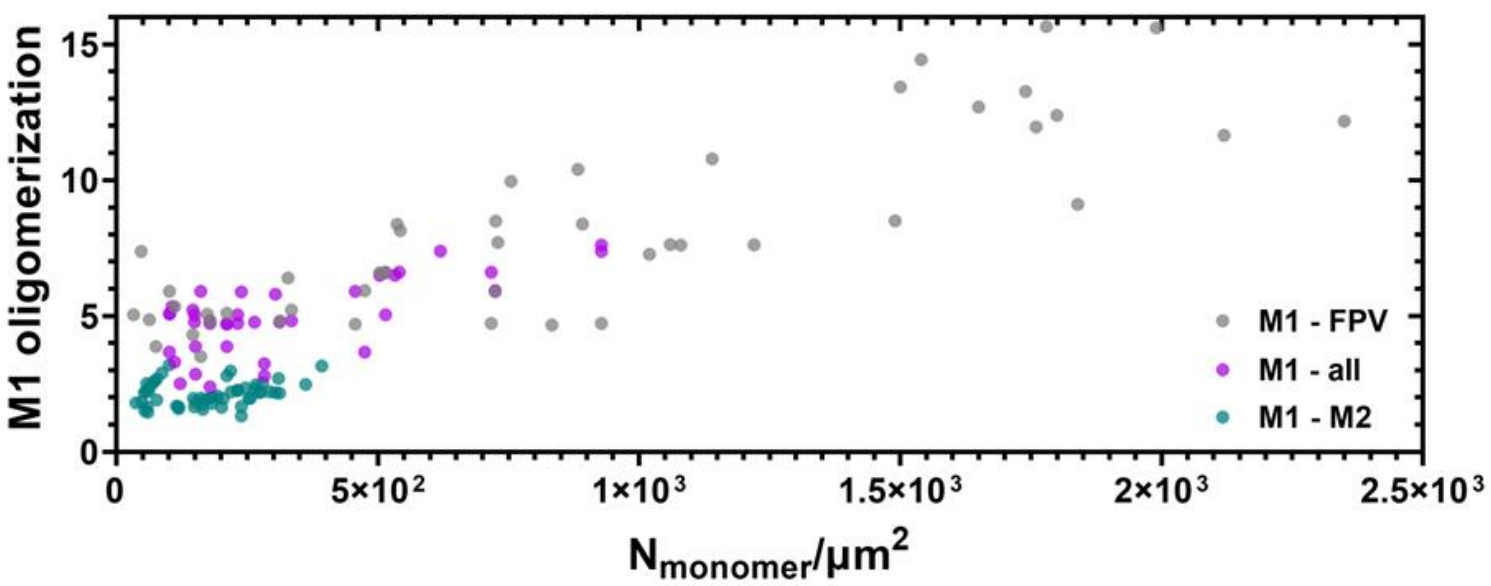

Figure 2: M1 oligomerizes in a concentration-dependent manner. Number and Brightness (N\&B) analysis of M1-mEGFP in cells expressing only M1-mEGFP, infected with FPV ("FPV"), co-transfected cells expressing unlabeled M2 and the reverse genetic plasmid system for all other FPV proteins ("all"), or co-transfected cells expressing unlabeled M2 ("M2"). Oligomerization and surface concentration values were obtained as described in the Methods section. A: Representative average intensity maps of M1-mEGFP in HEK293T cells. The average intensity map is visualized via color scale with units photon counts/dwell time. B: Representative brightness-intensity maps corresponding to the images represented in panel A. The images show pixel brightness as pixel color (counts/dwell time per molecule) and mean photon count rate as pixel intensity. C (left): Box plot of single data points from three independent experiments showing the normalized brightness (i.e. oligomerization) for M1-mEGFP and the corresponding controls (i.e., cytosolic monomer mEGFP (1x), cytosolic dimer mEGFP (2x) in the cytosol of HEK293T cells. C (right): Box plot of single data points from three independent experiments showing the 
oligomerization of M1-mEGFP at the PM of infected (M1-FPV) or co-transfected (M1-all, M1-M2) cells. Oligomerization values for PM-anchored controls (monomer mp-mEGFP(1x), dimer mp-mEGFP(2x)) are also shown. Sample size, median, and interquartile range (IQR) are indicated at the bottom. Horizontal dotted lines corresponding to oligomerization values $1,2,5$ and 7 are shown as guide to the eye. D: M1-mEGFP oligomerization as a function of surface concentration at the PM (in $\left.N_{\text {monomer }} / \mu^{2}\right)$. The number of measured cells were: M1-FPV $(n=46)$, M1-all $(n=39)$, and M1-M2 $(n=53)$.

Of note, it must be considered that in infected cells, M1 concentration and oligomerization are underestimated, due to the co-expression of viral unlabeled M1 which might take part in the formation of complexes with M1-mEGFP. Since N\&B analysis accounts only for labeled proteins, complexes containing both labeled and unlabeled species will effectively appear as smaller oligomers. Additionally, it is also possible that a precise determination of the multimeric state might be hindered by high protein concentrations at the PM, especially for very large multimers.

In summary, M1-mEGFP forms up to dimers in the cytoplasm or at the PM, upon co-expression of M2. The oligomerization of membrane-bound M1-mEGFP increases dramatically as a function of local concentration in infected cells and, to a minor extent, in cells expressing all other viral proteins via a reverse genetic plasmid system.

\section{HA and NA do not induce M1 oligomerization}

The interaction of $M 1$ with other viral membrane proteins (HA, NA, and $M 2$ ) is controversially discussed in previous studies $(10,11,22-24,30,31,35,36)$.

To clarify this issue, we performed 2-color sFCCS analysis in HEK293T cells expressing M1mEGFP in combination with i) mCherry2-M2, ii) mCherry2-HA $\mathrm{TMD}_{\mathrm{T}}$ and unlabeled $\mathrm{M} 2$, or iii) NAmCherry2 and unlabeled M2. As shown for example in Figure $3 \mathrm{~A}$ for the case of co-transfected cells expressing M1-mEGFP, mCherry2-HATMD, and unlabeled M2, M1 partitions strongly at the PM in all cases. For sFCCS measurements, the confocal detection volume is scanned in a linear fashion perpendicularly to the PM, as illustrated by the yellow arrow. Following the calculation of ACFs and CCFs, (Figure S7), this approach allows the quantification of the interactions between two differently labeled proteins by calculating the relative crosscorrelation (rel. cc), i.e. a measure of the relative abundance of molecular hetero-complexes. Furthermore, from the analysis of the ACF, SFCCS provides quantitative information about 
diffusion dynamics and, similar to N\&B analysis, the average oligomerization state of the monitored proteins.

Our results suggest that $\mathrm{M} 1$ forms monomers and dimers at the $\mathrm{PM}$, upon co-expression of $\mathrm{M} 2(1.7 \pm 0.8$, median $\pm I Q R, n=32$ cells $)$, confirming the results of the $N \& B$ experiments (Figure $3 \mathrm{~B}$ ). For comparison, the oligomerization state of control monomers (mp-mEGFP) and dimers (mp-mEGFP-mEGFP) is also shown. Further, the oligomerization of $M 1$ is not significantly altered by additionally co-expressing the IAV glycoproteins, mCherry2-HA $\mathrm{AMD}_{\text {TM }}(1.5$ \pm 0.6 , median \pm IQR, $n=46$ cells) or NA-mCherry2 ( $1.5 \pm 0.8$, median $\pm I Q R, n=36$ cells). To verify whether the FP fused to viral glycoproteins alters their quaternary structure, the molecular brightness of mCherry2-HATMD and NA-mCherry2 was also analyzed and compared to the corresponding controls (Figure $3 \mathrm{C}$ ). The HA transmembrane domain construct mCherry2-HATMD formed in average dimers $(2.1 \pm 0.6$, median $\pm I Q R, n=46$ cells), and NAmCherry2 formed in average tetramers ( $3.9 \pm 0.6$, median $\pm I Q R, n=36$ cells). Both oligomeric states are consistent with those obtained in earlier studies $(53,54)$. The average oligomerization state of mCherry2-M2 (3.3 \pm 1.0 , median $\pm I Q R, n=32$ cells) indicated that M2 might be present as a mixture of e.g. dimers and tetramers on the PM, which is consistent with previous results (55). Surprisingly, for all the examined IAV proteins, we observed that their average oligomerization state was not strongly influenced by their local concentration (Figure S8).

It is worth noting that the mCherry2-M2 construct (i.e., with mCherry2 fused to the $\mathrm{N}$ terminus of $\mathrm{M} 2$ ) was newly designed to monitor M1:M2 interactions while avoiding steric hindrance at the cytosolic side of $M 2$. In order to determine whether this fluorescent M2 construct behaves as expected (especially in the context of M1-M2 interactions), we used an alternative strategy to simultaneously express untagged $\mathrm{M} 2$ and a membrane marker ( $\mathrm{mp}$ mCherry2) via a bi-directional vector system (indicated as M2 $\leftrightarrow$ mp-mCherry2) (56). The measured concentration of mp-mCherry 2 can be used to estimate the amount of unlabeled M2 in the PM (see Materials and Methods in SI, Figure S9). The correct expression of M2 at the PM was validated by immunofluorescence (Figure S9 C). No significant difference in the oligomeric state of M1-mEGFP as a function of the surface concentration of M2 between both plasmid constructs (i.e., bidirectional M2 and mCherry2-M2) was observed (Figure S9 D). 
bioRxiv preprint doi: https://doi.org/10.1101/2021.05.06.442926; this version posted October 16, 2021. The copyright holder for this preprint (which was not certified by peer review) is the author/funder, who has granted bioRxiv a license to display the preprint in perpetuity. It is made available under aCC-BY-NC-ND 4.0 International license.

Therefore, only the mCherry2-M2 construct was used for further investigations of M1-M2 interaction.

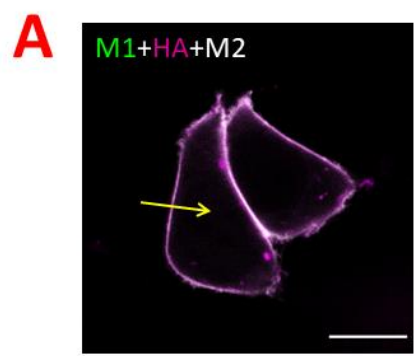

D

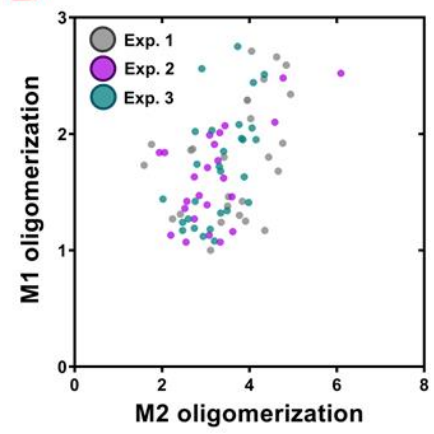

G

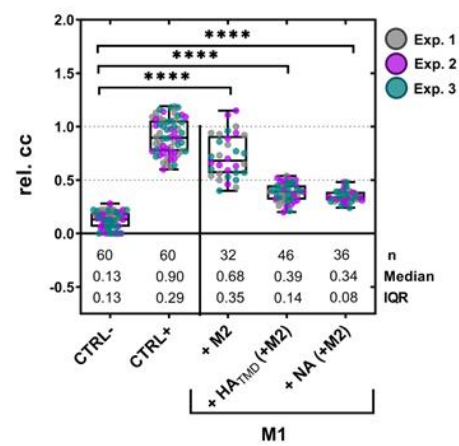

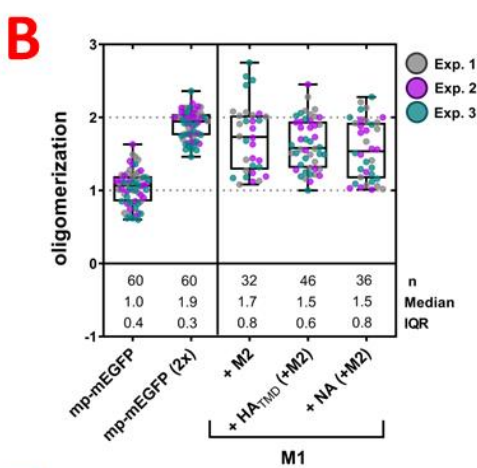

E

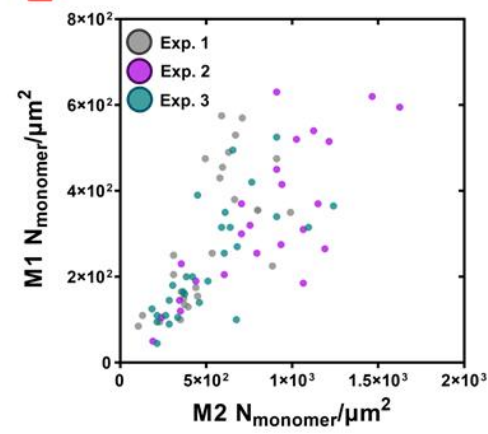

$\mathrm{H}$

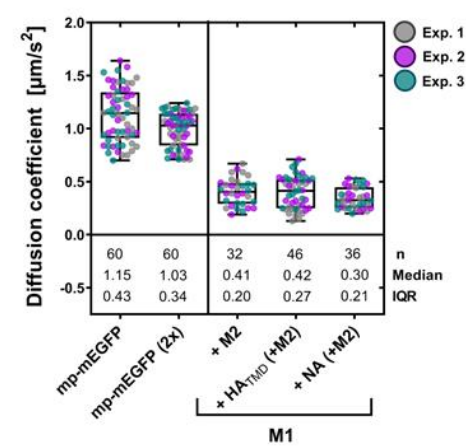

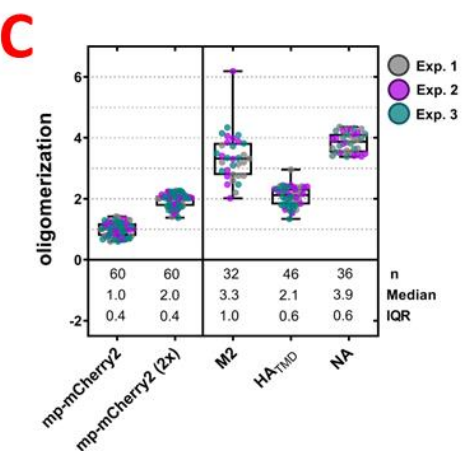

$\mathbf{F}$
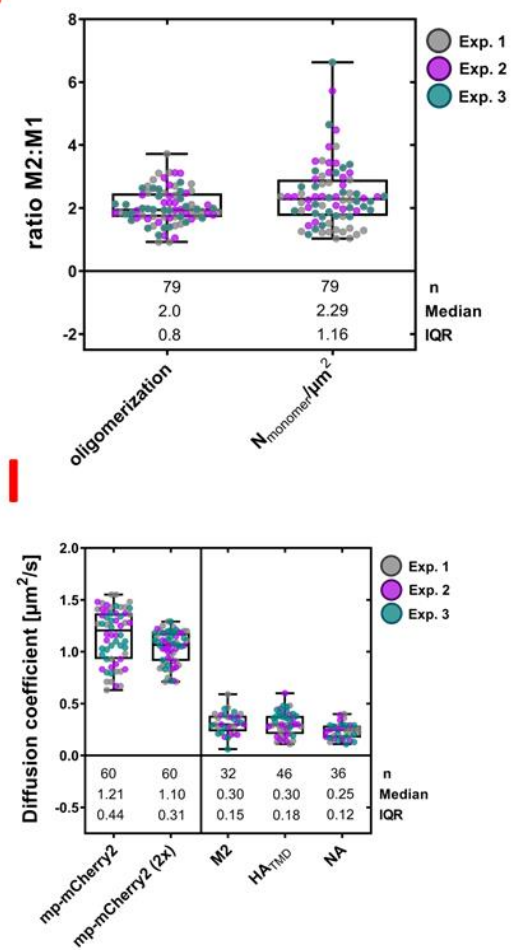

Figure 3: $M 2$ interacts with $M 1$ in a concentration-dependent manner. Scanning fluorescence cross-correlation spectroscopy (sFCCS) of M1-mEGFP in HEK293T cells co-expressing mCherry2-M2, mCherry2-HA $\mathrm{AMD}_{\mathrm{T}} / \mathrm{M} 2$-untagged, and NA-mCherry2/M2untagged. Oligomerization, surface concentration $\left(\mathrm{N}_{\text {monomer }} / \mu \mathrm{m}^{2}\right)$, cross-correlation, and diffusion coefficient $\left(\mu \mathrm{m}^{2} / \mathrm{s}\right)$ values were obtained as described in the Methods section. A: Representative confocal fluorescence image of HEK293T cells coexpressing M1-mEGFP (green), mCherry2-HA $A_{T M D}$ (magenta), M2-untagged. Yellow arrow indicates the scanning path used for sFCCS. Scale bar is $10 \mu \mathrm{m}$. B: Box plot with single data points from three independent experiments shows the oligomerization of the controls (monomer mp-mEGFP(1x) and dimer mp-mEGFP(2x)), and M1-mEGFP co-expressed with mCherry2-M2, mCherry2-HATMD /M2-untagged, and NA-mCherry2/M2-untagged. Sample size, median, and IQR are indicated in the graph. C: Box plot with single data points from three independent experiments shows the oligomerization of the controls (monomer $m p-m C h e r r y 2(1 x)$ and dimer $m p-m C h e r r y 2(2 x)$ ), and the viral surface proteins mCherry2-M2, mCherry2-HATMD, and NAmCherry2 for the same samples described for panel B. Sample size, median, and IQR are indicated in the graph. D-E: Scatter plots show the oligomerization of M1-mEGFP as a function of the oligomerization of mCherry2-M2 (D), and the surface concentration of M1-mEGFP as a function of the surface concentration of mCherry2-M2 (E). F: Box plot with single data points from three independent experiments shows the ratio of the oligomerization, and the surface concentration of M2:M1. Sample size, median, and IQR are indicated in the graph. G: Box plot with single data points from three independent 
experiments shows the relative cross-correlation (rel. cc) for the controls (negative control mp-mEGFP(1x)/mp-Cherry2 and positive control mp-mCherry2-mEGFP) and between M1-mEGFP and mCherry2-M2, mCherry2-HATMD, or NA-mCherry2. Cells expressing mCherry2-HATMD and NA-mCherry2 also expressed unlabeled M2. Sample size, median, and IQR are indicated in the graph. Statistical significance was determined using one-way ANOVA multiple comparison test (**** indicates $\mathrm{P}<0.0001$ compared to the negative control (CTRL-)). $\mathrm{H}$ : Box plot with single data points from three independent experiments shows the diffusion coefficient of the controls (monomer mp-mEGFP(1x) and dimer: mp-mEGFP(2x)), and M1-mEGFP co-expressed with mCherry2-M2, mCherry2-HA $A_{T M D} / \mathrm{M} 2$-untagged, and NA-mCherry2/M2-untagged. Sample size, median, and IQR are indicated in the graph. I: Box plot with single data points from three independent experiments shows the diffusion coefficient

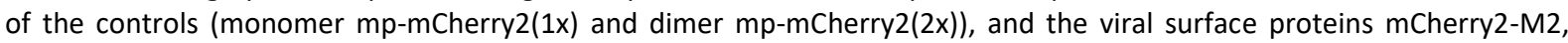
mCherry2-HATMD, and NA-mCherry2 for the same samples described for panel $\mathrm{H}$. Sample size, median, and IQR are indicated in the graph.

Notably, the oligomerization of M1-mEGFP was consistently independent from the concentration of mCherry2-M2 at the PM (Figure S9 D) but correlated with the oligomerization state of mCherry2-M2 (Figure $3 \mathrm{D}$ ). Also, the concentration of M1-mEGFP at the PM increased with increasing mCherry2-M2 concentration (Figure $3 \mathrm{E}$ ). As shown in Figure $3 \mathrm{~F}$, we could finally estimate that both M1-mEGFP concentration at the PM and oligomerization are circa half of what is observed for mCherry2-M2 (M2:M1 $1_{\text {oligo.state: }} 2.0 \pm 0.8$, and $\mathrm{M} 2: \mathrm{M} 1_{\text {surface conc.: }} 2.29$ \pm 1.16, median \pm IQR).

In summary, our results suggest that M1 binds to the PM as dimer upon co-expression of M2. M1-M1 and M1-lipid interactions did not appear to be modulated by the presence of HA or NA.

\section{M1 strongly interacts with M2 but only weakly associates to HA or NA}

Direct information regarding the formation of protein hetero-complexes at the PM can be derived by the analysis of ACFs and CCFs obtained via SFCCS (see previous paragraph). We therefore calculated the rel. cc as a measure of the hetero-interactions between M1-mEGFP and either mCherry2-M2, mCherry2-HA $A_{T M D}$, or NA-mCherry2 (Figure $3 \mathrm{G}$, and S3). Two interacting molecules diffusing together through the observation volume as a complex will give rise to a positive rel. cc that can be quantified by the amplitude of the cross-correlation curve (Figure S7 B). Low rel. cc indicates the absence of interaction between the observed proteins (see e.g., Figure S7 A). However, due to incomplete maturation of the fluorescent proteins and the partial overlap of the confocal volumes in both channels, the maximum achievable rel. cc value is lower than 1. For example, a tandem of mp-mCherry2-mEGFP used here as a positive control for rel. cc displayed a rel. cc of $0.90 \pm 0.29$ (median \pm IQR, $n=60$ 
cells). As expected, we detected a very low rel. cc (0.13 \pm 0.13 , median $\pm I Q R, n=60$ cells $)$ in negative control experiments (i.e., in samples of co-transfected cells expressing mp-mEGFP and mp-mCherry2). As shown in Figure $3 \mathrm{G}$, a rel. cc of $0.7 \pm 0.4$ (median \pm IQR, $n=32$ cells) was measured for M1-mEGFP and mCherry2-M2. This value is significantly higher than the negative control and close (ca. $80 \%$ ) to that obtained for the positive control, suggesting very strong association of M1-mEGFP with mCherry2-M2. Assuming a very simple scenario consisting e.g. of M1 dimers, M2 tetramers and 2:4 M1-M2 complexes, all detectable with $\mathrm{p}_{\mathrm{f}}=1$, ca. $80 \%$ of $\mathrm{M} 1$ molecules appear to be in complex with $\mathrm{M} 2$.

On the other hand, the obtained rel. cc values for M1-mEGFP with either mCherry2-HATMD, or NA-mCherry2 (rel. cc(M1,HATMD) $=0.39 \pm 0.14, n=46$ cells; rel. $c c(M 1, N A)=0.34 \pm 0.08, n=$ 36 cells, median \pm IQR) were lower but still significantly higher than the negative control. It is worth noting that such measurements could only be performed in the presence of unlabeled M2 since, without this third protein, no localization of M1-mEGFP at the PM could be observed (see previous paragraphs). The observed rel. cc values indicate a weak interaction between M1-mEGFP and the glycoproteins mCherry2-HATMD, and NA-mCherry2. In the simple approximation of $\mathrm{p}_{\mathrm{f}}=1$ and constant multimerization, independently from the participation in complexes, ca. $40 \%$ of $\mathrm{M} 1$ molecules appear to be associated with the viral glycoproteins. To further investigate this issue, we quantified the interaction between M1 and the glycoproteins also in infected cells. To this aim, we performed ccN\&B in cells infected with FPV and, additionally, co-transfected with M1-mEGFP and either mCherry2-HATMD or NA-mCherry2 plasmids. Similar to SFCCS, ccN\&B can be used to quantify the rel. cc between different FPs, especially in samples characterized by slow dynamics (52). Scanning FCCS measurements of M1-mEGFP in infected cells did not provide in fact reproducible results (data not shown). As shown in Figure S10, the rel. cc values determined by ccN\&B in infected cells for M1-mEGFP and $m$ Cherry2-HATMD (rel. $c c\left(M 1, H A_{T M D}\right)=0.31 \pm 0.10, n=21$ cells, median $\left.\pm I Q R\right)$, as well as for M1-mEGFP and NA-mCherry2 (rel. $c c(M 1, N A)=0.28 \pm 0.08, n=22$ cells, median $\pm I Q R$ ) were roughly comparable to the rel. cc values obtained in non-infected cells, as measured via sFCCS (Figure $3 \mathrm{G}$ ). A more precise quantification is complicated in this case by the presence of non-fluorescence viral proteins and unknown stoichiometry of the investigated molecular complexes. 
Finally, we quantified protein dynamics by fitting a two-dimensional diffusion model to the ACF data (Figure $3 \mathrm{H}$ and I, S7). Knowing the size of the observation volume, it is possible to obtain diffusion coefficients of the proteins ( $D$ in $\mu \mathrm{m} / \mathrm{s}^{2}$, see Materials and Methods). Protein diffusion depends in general on the size of the protein complex and on protein-membrane interactions. The diffusion coefficients measured for M1-mEGFP at the PM ( $D=0.3-0.4 \mu \mathrm{m} / \mathrm{s}^{2}$, Figure $3 \mathrm{H}$ ) were lower than those of the monomer control $\left(D=1.1 \pm 0.4 \mu \mathrm{m} / \mathrm{s}^{2}\right.$, median $\pm I Q R$, $n=60$ ), and similar to the diffusion coefficient of the IAV integral surface proteins mCherry2M2, mCherry2-HATMD, and NA-mCherry2 (indicated in Figure $3 \mathrm{I}$ ).

Taken together, our data indicate that M1 strongly interacts with M2. On the other hand, a relatively small amount of complexes containing M1 together with HA or NA was detected.

\section{Non-specific M1 recruitment to the PM is sufficient for the establishment of M1-HA interaction}

To investigate the origin of the interaction between M1 and HA (or NA) which was observed in cells additionally expressing $M 2$, we artificially induced $M 1$ binding to the PM. These experiments were performed to test the hypothesis that $M 1$ is recruited (by $M 2$ ) to the PM, where it can then interact with other membrane proteins (independently from the specific protein that first induced M1-PM binding).

Specifically, we designed two M1 constructs in which the protein was modified by myristoylation and palmitoylation (mp-M1-mEGFP) and, additionally, with a poly-lysine motif (mp-KrФ-M1-mEGFP), as shown in Figure $4 \mathrm{~A}$. The underlying idea is that the additional targeting sequences direct M1 specifically to lipid ordered "raft" domains (myristoyl-palmitoyl anchor (57)) or to regions containing acidic lipids (poly-lysine motif) in the PM, as supported by previous studies $(27,58-60)$. M1 localization within specialized PM domains might be indeed relevant, since the viral envelope proteins HA and NA were previously reported to localize in lipid "rafts", whereas M2 was observed at the edges between ordered and disordered domains $(7,14)$.

First, we verified the sub-localization of the two new constructs in transfected HEK293T cells. Both, mp-M1-mEGFP and mp-KrФ-M1-mEGFP, were efficiently trafficked to the PM (Figure 4 
B). Next, we examined the rel. cc between these two constructs and mCherry2-HATMD, as well as NA-mCherry2 (Figure $4 \mathrm{C}$ ) in co-transfected cells. The obtained rel. cc values (indicated in Figure 4 C) for mp-M1-mEGFP with mCherry2-HATMD or NA-mCherry2, as well as mp-KrФ-M1mEGFP with NA-mCherry2, were similar to those of the negative rel. cc control. These results indicate that NA-mCherry2 does not significantly interact with any of the modified membraneassociated M1 constructs. Also, mCherry2-HA $\mathrm{AMD}_{T M}$ does not seem to interact with the supposedly lipid raft-associated mp-M1-mEGFP. In contrast, a reproducible interaction between mp-KrФ-M1-mEGFP and mCherry2-HA $A_{T M D}\left(\right.$ rel. cc $\left(m p-K r \Phi-M 1, H A_{T M D}\right)=0.26 \pm 0.18$, $\mathrm{n}=30$ cells, median \pm IQR) was observed. Notably, the rel. cc value observed in this case was significantly lower than the one obtained in the context of the interaction between (wildtype) M1-mEGFP and mCherry2-HATMD, in the presence of M2. Next, we calculated the surface concentration of each protein and plotted the cross-correlation values against the surface concentration, as well as the ratio of the concentration between the protein pairs (Figure S11). This analysis was performed to exclude that the obtained rel. cc values are influenced by the surface concentration of the proteins or the expression ratio between the proteins. No concentration-dependency of the rel. cc for all pairs was observed.

Finally, we quantified the diffusion dynamics of the examined protein constructs (Figure 4 D). The obtained diffusion coefficient values (shown in Figure $4 \mathrm{D}$ ) for mp-M1-mEGFP in the presence of mCherry2-HA $\mathrm{AMD}_{T M}$ or NA-mCherry2 were similar to those of the monomer control (mp-mEGFP). A similar observation was made for mp-KrФ-M1-mEGFP in the presence of NAmCherry2. The fact that these M1 constructs diffuse as fast as a lipid-anchored protein (rather than a membrane-spanning protein, see Figure 3 I) suggests the absence of significant interactions/co-diffusion of M1 with mCherry2-HA $\mathrm{AMD}_{\mathrm{T}}$ or NA-mCherry2. For comparison, the diffusion coefficients of M1-mEGFP in the presence of $M 2$ and one glycoprotein are also reported in Figure $4 D\left(D=0.38 \pm 0.23 \mu \mathrm{m} / \mathrm{s}^{2}\right.$, median $\pm I Q R, n=46$, when co-expressed e.g. with mCherry2-HA $\left.A_{T M D}\right)$. This result is comparable to the diffusion coefficient of mCherry2-M2 $\left(D=0.30 \pm 0.15 \mu \mathrm{m} / \mathrm{s}^{2}\right.$, median $\pm I Q R, n=46$, Figure $\left.3 \mathrm{I}\right)$. Interestingly, the diffusion coefficient for mp-KrФ-M1-mEGFP ( $D=0.60 \pm 0.16 \mu \mathrm{m} / \mathrm{s}^{2}$, median $\pm I Q R, \mathrm{n}=32$ ) co-expressed with mCherry2-HАTMD was slightly lower than that measured for the monomer control, although still higher than the one measured for M1-mEGFP in the presence of unlabeled M2. It is also worth noting that the distribution of diffusion coefficient values for the above-mentioned 
bioRxiv preprint doi: https://doi.org/10.1101/2021.05.06.442926; this version posted October 16, 2021. The copyright holder for this preprint (which was not certified by peer review) is the author/funder, who has granted bioRxiv a license to display the preprint in perpetuity. It is made available under aCC-BY-NC-ND 4.0 International license.

sample appears to slightly deviate from a normal distribution (Kolmogorov-Smirnov test P value $=0.0356$ ). The reason for this deviation is not clear at this point but one possible cause might be, for example, the occasional presence of cytosolic signal (see e.g. Figure 4 B) weakly interfering with measurements at the PM.

In conclusion, NA-mCherry2 does not exhibit significant cross-correlation or co-diffusion with neither of the "artificially" PM-associated M1 proteins. In contrast, mCherry2-HA TMD appears to interact with M1 depending on the specific way in which the latter is anchored to the PM.
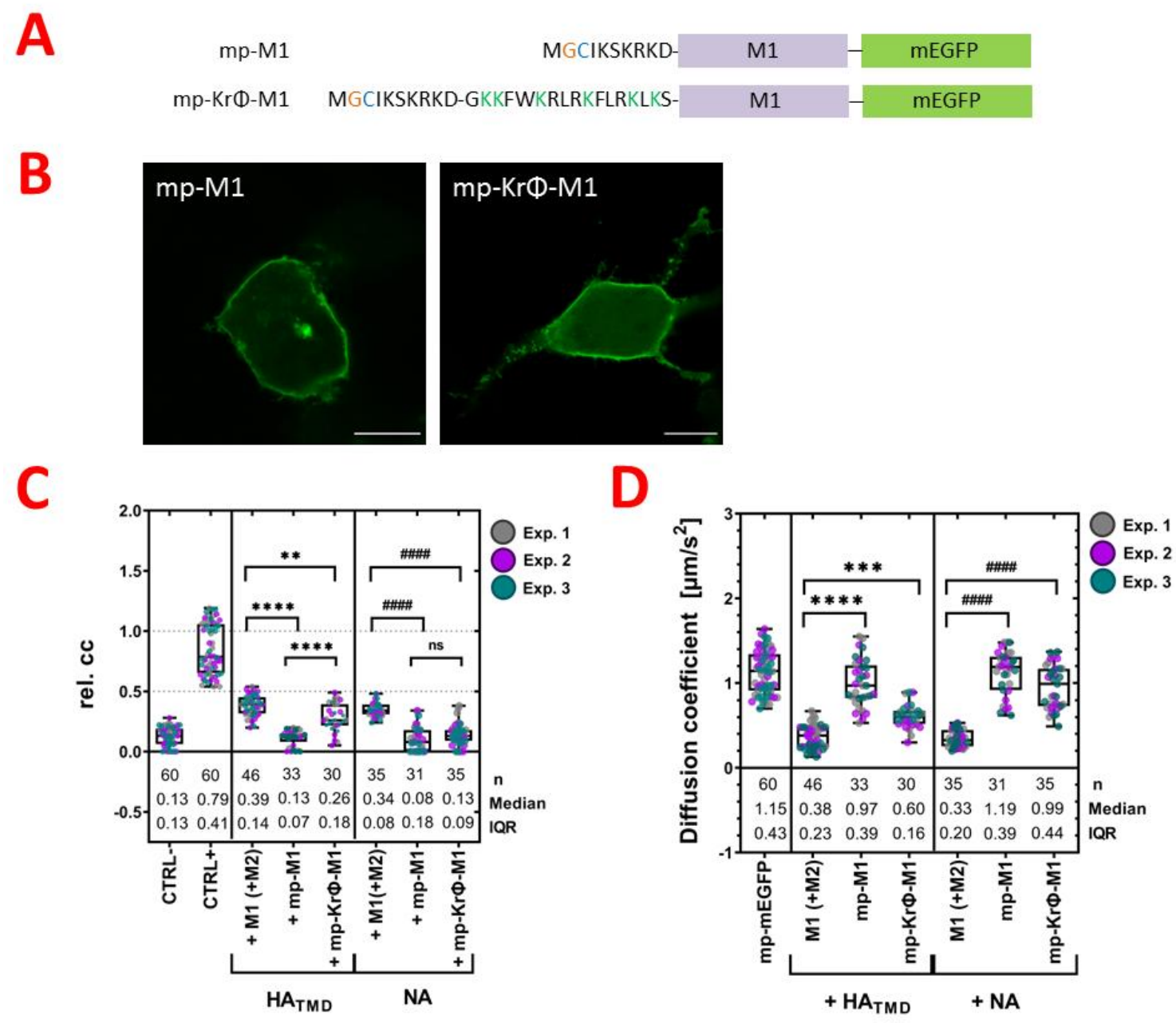

Figure 4: HA interacts with a membrane-associated M1 construct. A: Schematic diagram of $\mathrm{M} 1$ constructs with $\mathrm{N}$-terminal PM-targeting sequences. One construct has a myristoylation (orange) and palmitoylation (blue) motif (mp-M1-mEGFP), and the other on has an additional poly-lysine motif (green letters, mp-KrФ-M1-mEGFP). B: Representative M1 subcellular localization images in transfected HEK293T cells expressing mp-M1-mEGFP (left side), or mp-KrФ-M1-mEGFP (right side). Scale bars represent $10 \mu \mathrm{m}$. C: Box plot with single data points from three independent experiments shows the crosscorrelation for the controls (negative control mp-mEGFP(1x)/mp-mCherry2(1x) and positive control mp-mCherry2-mEGFP), and between M1-mEGFP (or mp-M1-mEGFP, or mp-KrФ-M1-mEGFP) and mCherry2-HA $A_{T M D}$, or NA-mCherry2. Sample size, median, and IQR are indicated in the graph. Statistical significance was determined using one-way ANOVA multiple comparison test $(* *$ indicates $p<0.01, * * * *$ indicates $p<0.0001$ compared to M1-mEGFP/ mCherry2-HA TMD; \#\#\# indicates $p$ 
< 0.0001 compared to M1-mEGFP/ NA-mCherry2, ns indicates not significant). D: Box plot with single data points from three independent experiments shows the diffusion coefficient of the monomer control (mp-mEGFP), and M1-mEGFP, mp-M1mEGFP, and mp-KrФ-M1-mEGFP co-expressed with mCherry2-HA ${ }_{T M D}$, or NA-mCherry2. Sample size, median, and IQR are indicated in the graph. Statistical significance was determined using one-way ANOVA multiple comparison test (*** indicates $\mathrm{p}<0.001, * * * *$ indicates $\mathrm{p}<0.0001$ compared to M1-mEGFP/ mCherry2-HA $\mathrm{TMD}_{\mathrm{TM}}$; \#\#\# indicates $\mathrm{p}<0.0001$ compared to M1mEGFP/ NA-mCherry2).

\section{A potential M2 binding site is located in the N-domain (aa 1-67) of M1.}

An interaction site for $\mathrm{M} 2$ has not been yet identified within $\mathrm{M} 1$. Therefore, we created different M1 constructs for the expression of specific protein subdomains, in order to locate a potential $\mathrm{M} 2$ binding site (Figure $5 \mathrm{~A}$ ). The truncated $\mathrm{M} 1$ constructs encoded $\mathrm{i}$ ) the $\mathrm{N}$ - and $\mathrm{M}$ M-domains (NM1, amino acids 1-164), ii) the $\mathrm{N}$-terminus domain including the linker region (NM1, amino acids 1-86), iii) only the N-domain (NM1, amino acids 1-67) or iv) the M1 Cdomain (CM1, amino acids 165-252). A mEGFP was fused to the C-terminal site of each M1 variants. Moreover, a well conserved amino acid sequence in the cytoplasmic C-terminal tail of $\mathrm{M} 2$ at the position 71 and 73 was previously shown as an interaction site for M1 (10). Hence, we generated a substitution mutant of $\mathrm{M} 2(\mathrm{M} 2 \mathrm{mut})$ in which the triplet sequence (71 - SMR 73) was replaced by alanine (Figure $5 \mathrm{~A}$ ).

To verify whether the truncated M1-mEGFP constructs are altered in their subcellular localization, we observed them in HEK293T in the absence of mCherry2-M2. All truncated M1mEGFP variants showed a similar subcellular localization to the wildtype M1-mEGFP (Figure 1 $\mathrm{A}$, and $5 \mathrm{~B}$ ). Next, all truncated M1-mEGFP constructs were co-expressed with mCherry2-M2 in HEK293T cells. All N-terminus M1 variants were recruited to the PM whereas the C-terminus M1 construct showed still a homogeneous distribution in the cytoplasm (Figure $5 \mathrm{C}$ ). The percentages of cells with $\mathrm{M} 1$ at the PM for the NM1 variants were similar as observed for the $\mathrm{M} 1$ wildtype in co-expression with mCherry2-M2 (Figure S4 C). These results indicated that the $\mathrm{M} 2$ binding site might be pinpointed to the $\mathrm{N}$-terminal domain of $\mathrm{M} 1$ and, specifically, to the amino acids 1-67. Furthermore, no recruitment of M1 wildtype was observed upon a coexpression with the mCherry2-M2 mut (Figure $5 \mathrm{D}$ ). Based on this result, we could confirm that the recruitment of M1 to PM occurred via a specific interaction of M1 with the amino acid sequence $(71-S M R-73)$ on $M 2$. 
bioRxiv preprint doi: https://doi.org/10.1101/2021.05.06.442926; this version posted October 16, 2021. The copyright holder for this preprint (which was not certified by peer review) is the author/funder, who has granted bioRxiv a license to display the preprint in perpetuity. It is made available under aCC-BY-NC-ND 4.0 International license.

\begin{tabular}{|c|c|c|c|c|c|c|}
\hline & & & 7 & & & \\
\hline$M 1_{w t}$ & $\mathrm{NH} 2-$ & $\mathrm{N}$-domain & linker & M-domain & C-domain & mEGFP \\
\hline $\mathrm{NM1}_{1-67}$ & $\mathrm{NH} 2-$ & $\mathrm{N}$-domain & & & & mEGFP \\
\hline $\mathrm{NM1}_{1-86}$ & $\mathrm{NH} 2-$ & $\mathrm{N}$-domain & linker & & & mEGFP \\
\hline $\mathrm{NM1}_{1-164}$ & $\mathrm{NH} 2-$ & $\mathrm{N}$-domain & linker & M-domain & & mEGFP \\
\hline $\mathrm{CM1}_{165-252}$ & $\mathrm{NH} 2-$ & 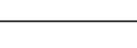 & & & C-domain & mEGFP \\
\hline
\end{tabular}

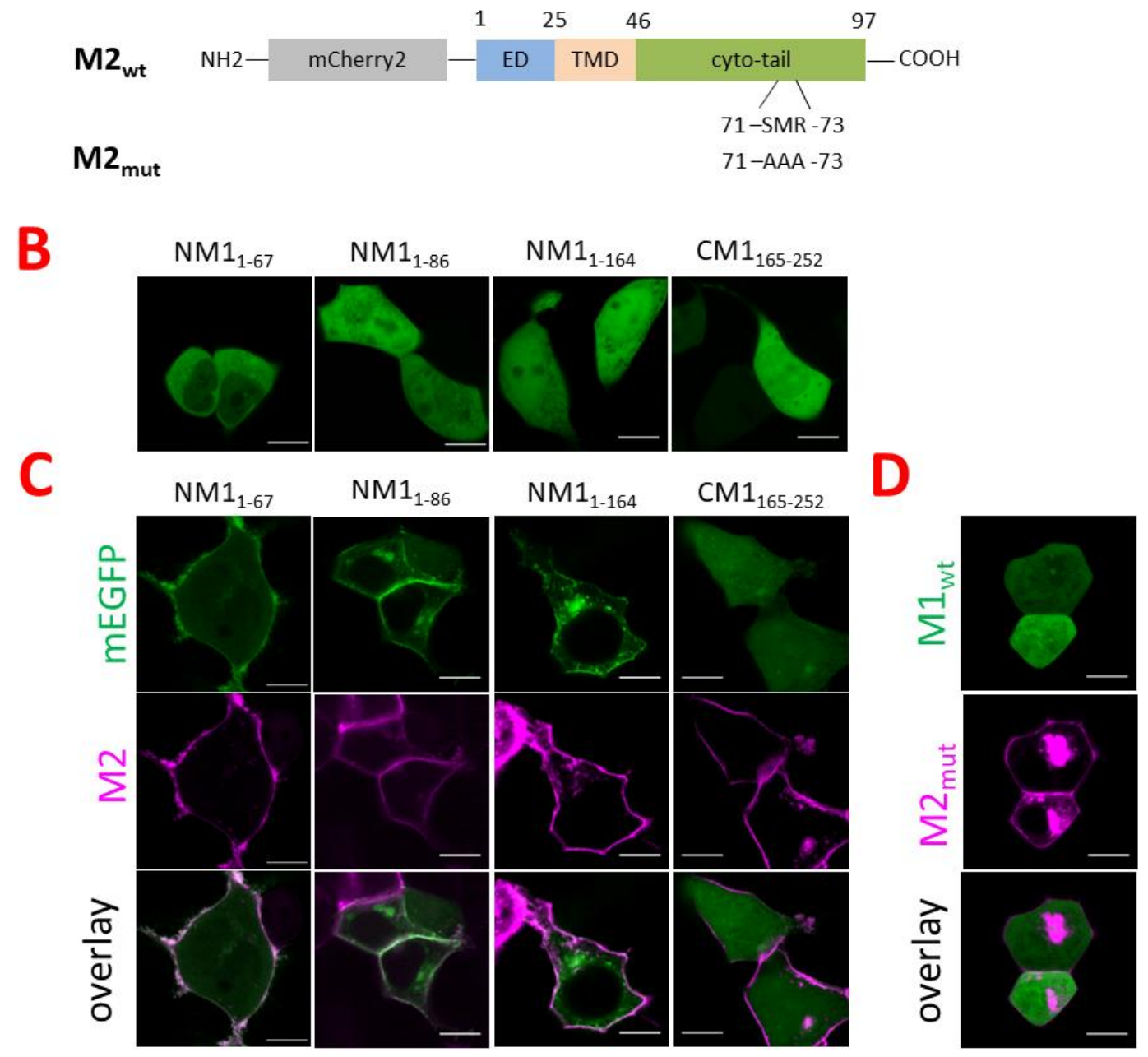

Figure 5: $\mathrm{M} 2$ binding site on $\mathrm{M} 1$ is located in the $\mathbf{N}$-terminus domain. A: Schematic diagram of different $\mathrm{M} 1$ and $\mathrm{M} 2$ expression constructs. On top, M1 constructs showing the wildtype and the truncated $\mathrm{M} 1$ variants with their domains: $\mathrm{N}$ terminus domain ( $\mathrm{N}$-domain, aa 1-67, blue), linker region (orange, aa 68-86), middle domain (M-domain, aa 87-164, green), and C-terminus domain (C-domain, aa 165-252, purple). A mEGFP was fused to the C-terminus of each protein construct. On the bottom, M2 constructs showing the wildtype and the M2 mutant (71-SMR-73 was replaced by three alanine) with their domains: ectodomain (ED, aa 1-25, blue), transmembrane domain (TMD, aa 26-46, orange), cytoplasmic tail (cyto-tail, aa 4797, green). Each construct had a mCherry2 fused to the N-terminal site of M2. B: Representative confocal fluorescence images of HEK293T cells expressing truncated M1-mEGFP variants: $\mathrm{NM1}_{1-67}, \mathrm{NM1}_{1-86}, \mathrm{NM1}_{1-164}$, and $\mathrm{CM} 1_{165-252}$. C: Representative confocal fluorescence images of HEK293T cells expressing truncated M1-mEGFP variants: $\mathrm{NM} 1_{1-67}, \mathrm{NM} 1_{1-86}, \mathrm{NM} 1_{1-164}$, and $\mathrm{CM}_{165-252}$ (green) in the presence of wildtype mCherry2-M2 (magenta). (D) Representative confocal fluorescence images of HEK293T cells expressing wildtype M1-mEGFP (green) with mCherry2-M2 mutant (M2 mut, magenta). Scale bars represent 10 $\mu \mathrm{m}$. 


\section{Discussion}

The role of M1 in IAV assembly is of fundamental importance, as it is now understood that this protein connects together the viral envelope, its membrane proteins ( $H A, N A$, and $M 2$ ) and the genome (61). It has been suggested that interactions of M1 with the viral glycoproteins (e.g. HA) drive M1 localization to the PM of infected cells (10, 11, 22-24), but other studies reported conflicting data regarding the interaction of M1 with HA and NA (30, $31,35,36)$. Such findings are mostly based on biochemistry approaches providing indirect interaction data $(7,62)$.

Therefore, in order to quantify protein-protein interactions directly at the PM of living cells, we performed fluorescence fluctuation spectroscopy experiments under physiological conditions. Such experimental approaches (i.e., $\mathrm{sF}(\mathrm{C}) \mathrm{CS}$ and (cc)N\&B) provide information regarding the oligomeric state, surface concentration, hetero-interactions and dynamics in complex biological systems $(51,52,63-66)$.

To this aim, we selected HEK293T cells as a cellular model because they are often used for reverse genetic virus production $(39,40,67)$ and were shown to be appropriate for IAV protein expression $(17,41,42)$. Additionally, we produced and tested several fluorescent IAV protein constructs. Of note, the fluorescent NA construct designed in this study allowed for the first time the investigation of the interaction between this IAV glycoprotein and other viral proteins directly in living cells. It is worth noting that incorporating fluorescent fusion tags might have an impact in general on the localization, function, and conformation of the protein of interest $(68,69)$. For example, our control experiments showed that the cellular distribution of M1 with an mEGFP fused to its C-terminus was similar to that of unlabeled M1 $(46,47)$, whereas an N-terminally fused mEGFP M1 variant seemed to have transport failures which are probably caused by steric hindrance between fluorophore and signal peptide (47). On the other hand, the fluorescent constructs used to investigate the viral envelope proteins (HA, NA, and $\mathrm{M} 2$ ) were all localized at the PM, similar to the corresponding non-fluorescent proteins $(48,49)$, and yielded the expected oligomerization state $(41,42,53,54)$. For example, our results are compatible with the presence of NA tetramers and mixtures of $M 2$ dimers and tetramers (Figure $3 \mathrm{C}$ ), in agreement with previous data $(55,70)$. Furthermore, we also demonstrated that the protein-protein interactions investigated here (e.g. between M1- 
mEGFP and mCherry2-M2) are specific and analogous to those observed in other contexts (10), as shown by mutagenesis experiments (Figure 5 D) and using unlabeled interaction partners (Figure S1).

In order to identify the minimum requirement for $\mathrm{M} 1$ association to the $\mathrm{PM}$, we observed cells expressing different combinations of viral proteins. First, we confirmed that M1 does not bind to the PM in the absence of other viral proteins, despite the observation of strong lipid-protein interactions previously observed in model membrane systems (17-20). Surprisingly, we did not observe any recruitment of M1 to the PM in the presence of HA or NA (Figure 1). It is worth noting that several studies proposed that M1 interacts with the cytoplasmic tails of HA or NA $(23,30,71-74)$, but our direct observations in living cells strongly suggest that the two IAV glycoproteins are not able to recruit M1 to the PM by themselves. It is unlikely that the lack of interaction might be a simple consequence of the presence of fluorescent labels, since $\mathrm{HA}$ and NA are labeled at the extracellular side. Also, the same M1-mEGFP strongly associates with the PM in the presence of M2. This result is in agreement with previous studies indicating that M1-M2 interactions affect M1 localization and drive virus assembly $(10,11,27,75,76)$. For the first time, we could provide direct experimental evidence that the M2-binding region is located within the first 67 amino acids of M1 (Figure 5). Also, thanks to the application of quantitative fluorescence microscopy methods, we could additionally prove that M1 and M2 do not simply colocalize at the PM but rather form complexes. This conclusion is supported by the similar diffusion dynamics observed for $\mathrm{M} 1$ and $\mathrm{M} 2$ (i.e. diffusion coefficients typical of trans-membrane proteins rather than membrane-associated proteins, Figure $3 \mathrm{H}$ ) and by the significant degree of cross-correlation between the signals of the two proteins (Figure $3 \mathrm{G}$ ). Due to the lack of strong intracellular co-localization (Figure $1 \mathrm{C}$ and $5 \mathrm{C}$ ), we hypothesize that M1 diffuses freely in the cytoplasm and M1-M2 interaction occurs directly at the PM. M1-M2 complexes appear to consist, in average, of 1-2 M1 and 2-4 M2 molecules (Figure 3). Assuming that each $M 2$ monomer has a binding site for $M 1$, the observed 1:2 stoichiometry suggests that the M1 binding might be limited for example by steric constraints or competition with other binding partners of M2 (e.g., LC3 (42)). Furthermore, in the simple approximation of M1 dimers, M2 tetramers, and 2:4 M1-M2 complexes being associated to the PM, our crosscorrelation measurements indicate that ca. $80 \%$ of M1 is indeed complexed to M2. The remaining amount of PM-associated M1 might interact with e.g. acidic lipids at the PM (19, 
20) but, of note, we never observed any significant degree of M1 localization at the PM in the absence of M2. This finding puts forward the hypothesis that M2-M1 complex formation might facilitate the interaction of M1 with other membrane components. This mechanism might also explain previous findings indicating the presence of $\mathrm{HA}$ and $\mathrm{M} 1$ in the same membrane fractions $(22,23)$ or within the same region in the PM $(15)$. Accordingly, we have observed that in the presence of M2 (i.e. M1 being efficiently recruited to the PM) there is a significant (although modest) interaction between M1 and the glycoproteins HA or NA (Figure $3 \mathrm{G}$ ). On one hand, it is possible that e.g. M1-HA interactions are not direct but, rather, mediated by $M 2$ (15). Alternatively, it is possible that, while $M 2$ is needed for the initial recruitment of $M 1$ to the PM, M1-M2 interactions are not long-lived and can be partially replaced for example by M1-HA interactions. In this case, M2 might induce interactions between M1 and other membrane components by e.g. increasing M1 local concentration in specific PM regions or stabilizing a certain geometric configuration of M1. Based on control experiments monitoring M1-HA/NA interactions as a function of local protein concentration (Figure S11), a prominent role of concentration seems unlikely though. To evaluate whether $M 2$ is strictly needed for HA-M1 interactions, we performed sFCCS experiments in which M1 was artificially anchored to the PM (Figure 4). In this case, depending on the specific lipid anchor, we were able to observe M1-HA interactions also in the absence of $\mathrm{M} 2$, thus indicating that $i$ ) the latter protein is not always required as a bridge between M1 and IAV glycoproteins and ii) the lipid environment plays a role in the establishment of interactions among IAV proteins. Of interest, it was shown that HA is associated to specific lipids, such as $\operatorname{PI}(4,5) \operatorname{P} 2(14,49)$ and this observation might provide a molecular mechanism explaining our observation of nonnegligible M1-HA interactions, in the case that M1 was artificially anchored to the membrane via lipidation and, additionally, a polybasic motif. The degree of association between HA and mp-KrФ-M1-mEGFP appeared to be between that observed for wt M1 and that observed for mp-M1-mEGFP, as supported by the observation of intermediate cross-correlation values (Figure $4 \mathrm{C}$ ) and diffusion dynamics (Figure $4 \mathrm{D}$ ).

The observation that one single IAV membrane protein (i.e. M2) is sufficient for the recruitment of $\mathrm{M} 1$ to the $\mathrm{PM}$ prompted us to investigate whether M1-M2 interaction is also sufficient for the initiation of the large-scale M1 multimerization associated with IAV assembly (17). Our experiments clearly demonstrate that this is not the case, since M1 remains, in 
average, mostly dimeric when bound to the PM in the presence of M2 (Figure $2 \mathrm{C}$ ). On the other hand, in the presence of all the other viral proteins, M1 formed larger multimers (up to 5-10 monomers). This effect does not seem to be a direct consequence of the presence of HA or NA alone (Figure $3 \mathrm{~B}$ ) and is even stronger in infected cells. It is worth noting that the formation of very large multimers of M1-mEGFP in infected cells might be partially due to i) higher M1 concentrations at the PM or ii) the presence of unlabeled M1 molecules which more efficiently support protein-protein interactions. It was in fact reported that fluorescent viral proteins might not be able to oligomerize on a very large scale (65). Alternatively, other viral proteins or altered lipid metabolism (and, consequently, modification of PM composition) in infected cells might play a role and these possibilities are currently under investigation.

In conclusion, our study sheds light on the very first steps in IAV assembly. According to our results, the main role of $M 2$ in this context is to recruit $M 1$ to specific regions of the PM. This is in agreement with previously proposed models according to which M2 chaperones M1 to the PM (77) and, specifically, to interface regions between "raft" and "non-raft" domains (14, 16) or domains enriched in negatively-charged lipids (17). In further steps, M1 can then interact with lipids and other viral proteins and such interactions might be involved in the formation of larger protein complexes eventually leading to IAV capsid assembly.

\section{Author contributions}

Research planning, A.P. and S.C; investigation, A.P.; data analysis, A.P.; writing-original draft preparation, A.P. and S.C.; writing-review and editing, V.D. and S.B.; software, V.D. and S.C.; supervision, S.C.; funding acquisition, S.C.

\section{Acknowledgments}

This work was supported by the German Research Foundation (DFG grant \#254850309 to S.C.). The authors thank Andreas Herrmann for reading the manuscript and providing useful feedback.

\section{Supporting citations}

References (78-86) appear in the Supporting Information. 


\section{Reference}

1. Davlin, S., L. Blanton, K. Kniss, D. Mustaquim, S. Smith, N. Kramer, J. Cohen, C. Cummings, S. Garg, B. Flannery, A. Fry, L. Grohskopf, J. Bresee, T. Wallis, W. Sessions, R. Garten, X. Xu, A. Elal, L. Gubareva, and L. Brammer. 2016. Influenza Activity - United States, 2015-16 Season and Composition of the 2016-17 Influenza Vaccine. MMWR. Morbidity and mortality weekly report 65:567-575.

2. Taubenberger, J. K., and D. M. Morens. 2009. Pandemic influenza--including a risk assessment of H5N1. Rev Sci Tech 28:187-202.

3. Bouvier, N. M., and P. Palese. 2008. The biology of influenza viruses. Vaccine 26 Suppl 4:D49-D53.

4. Taubenberger, J. K., and J. C. Kash. 2010. Influenza virus evolution, host adaptation, and pandemic formation. Cell Host Microbe 7:440-451.

5. Mostafa, A., E. M. Abdelwhab, T. C. Mettenleiter, and S. Pleschka. 2018. Zoonotic Potential of Influenza A Viruses: A Comprehensive Overview. Viruses 10:497.

6. Samji, T. 2009. Influenza A: understanding the viral life cycle. Yale J Biol Med 82:153159.

7. Veit, M., and B. Thaa. 2011. Association of influenza virus proteins with membrane rafts. Adv Virol 2011:370606.

8. Dou, D., R. Revol, H. Östbye, H. Wang, and R. Daniels. 2018. Influenza A Virus Cell Entry, Replication, Virion Assembly and Movement. Front Immunol 9:1581.

9. Manzoor, R., M. Igarashi, and A. Takada. 2017. Influenza A Virus M2 Protein: Roles from Ingress to Egress. Int J Mol Sci 18.

10. Chen, B. J., G. P. Leser, D. Jackson, and R. A. Lamb. 2008. The influenza virus M2 protein cytoplasmic tail interacts with the M1 protein and influences virus assembly at the site of virus budding. Journal of virology 82:10059-10070.

11. McCown, M. F., and A. Pekosz. 2006. Distinct domains of the influenza a virus M2 protein cytoplasmic tail mediate binding to the M1 protein and facilitate infectious virus production. Journal of virology 80:8178-8189.

12. Rossman, J. S., X. Jing, G. P. Leser, V. Balannik, L. H. Pinto, and R. A. Lamb. 2010. Influenza virus $\mathrm{m} 2$ ion channel protein is necessary for filamentous virion formation. Journal of virology 84:5078-5088.

13. Rossman, J. S., X. Jing, G. P. Leser, and R. A. Lamb. 2010. Influenza virus M2 protein mediates ESCRT-independent membrane scission. Cell 142:902-913.

14. Veit, M., S. Engel, B. Thaa, S. Scolari, and A. Herrmann. 2013. Lipid domain association of influenza virus proteins detected by dynamic fluorescence microscopy techniques. Cell Microbiol 15:179-189.

15. Leser, G. P., and R. A. Lamb. 2017. Lateral Organization of Influenza Virus Proteins in the Budozone Region of the Plasma Membrane. J Virol 91.

16. Rossman, J. S., and R. A. Lamb. 2011. Influenza virus assembly and budding. Virology 411:229-236.

17. Bobone, S., M. Hilsch, J. Storm, V. Dunsing, A. Herrmann, and S. Chiantia. 2017. Phosphatidylserine Lateral Organization Influences the Interaction of Influenza Virus Matrix Protein 1 with Lipid Membranes. J Virol 91:e00267-00217.

18. Dahmani, I., K. Ludwig, and S. Chiantia. 2019. Influenza A matrix protein M1 induces lipid membrane deformation via protein multimerization. Biosci Rep 39. 
19. Hilsch, M., B. Goldenbogen, C. Sieben, C. T. Höfer, J. P. Rabe, E. Klipp, A. Herrmann, and S. Chiantia. 2014. Influenza A matrix protein M1 multimerizes upon binding to lipid membranes. Biophys J 107:912-923.

20. Höfer, C. T., S. Di Lella, I. Dahmani, N. Jungnick, N. Bordag, S. Bobone, Q. Huang, S. Keller, A. Herrmann, and S. Chiantia. 2019. Structural determinants of the interaction between influenza $A$ virus matrix protein $\mathrm{M} 1$ and lipid membranes. Biochim Biophys Acta Biomembr 1861:1123-1134.

21. Noton, S. L., E. Medcalf, D. Fisher, A. E. Mullin, D. Elton, and P. Digard. 2007. Identification of the domains of the influenza A virus M1 matrix protein required for NP binding, oligomerization and incorporation into virions. J Gen Virol 88:2280-2290.

22. Barman, S., A. Ali, E. K. W. Hui, L. Adhikary, and D. P. Nayak. 2001. Transport of viral proteins to the apical membranes and interaction of matrix protein with glycoproteins in the assembly of influenza viruses. Virus Research 77:61-69.

23. Jin, H., G. P. Leser, J. Zhang, and R. A. Lamb. 1997. Influenza virus hemagglutinin and neuraminidase cytoplasmic tails control particle shape. EMBO J 16:1236-1247.

24. Zhang, J., A. Pekosz, and R. A. Lamb. 2000. Influenza virus assembly and lipid raft microdomains: a role for the cytoplasmic tails of the spike glycoproteins. Journal of virology 74:4634-4644.

25. Darapaneni, V. 2015. Large-scale Analysis of Influenza A Virus Sequences Reveals Universally Conserved Residues of Matrix Proteins. American Journal of Current Microbiology 3:1-13.

26. Nayak, D. P., R. A. Balogun, H. Yamada, Z. H. Zhou, and S. Barman. 2009. Influenza virus morphogenesis and budding. Virus Res 143:147-161.

27. Wang, D., A. Harmon, J. Jin, D. H. Francis, J. Christopher-Hennings, E. Nelson, R. C. Montelaro, and F. Li. 2010. The Lack of an Inherent Membrane Targeting Signal Is Responsible for the Failure of the Matrix (M1) Protein of Influenza A Virus To Bud into Virus-Like Particles. Journal of Virology 84:4673.

28. Ruigrok, R. W., A. Barge, P. Durrer, J. Brunner, K. Ma, and G. R. Whittaker. 2000. Membrane interaction of influenza virus M1 protein. Virology 267:289-298.

29. Baudin, F., I. Petit, W. Weissenhorn, and R. W. Ruigrok. 2001. In vitro dissection of the membrane and RNP binding activities of influenza virus M1 protein. Virology 281:102108.

30. Ali, A., R. T. Avalos, E. Ponimaskin, and D. P. Nayak. 2000. Influenza virus assembly: effect of influenza virus glycoproteins on the membrane association of M1 protein. Journal of virology 74:8709-8719.

31. Enami, M., and K. Enami. 1996. Influenza virus hemagglutinin and neuraminidase glycoproteins stimulate the membrane association of the matrix protein. Journal of virology 70:6653-6657.

32. Su, W.-C., W.-Y. Yu, S.-H. Huang, and M. M. C. Lai. 2018. Ubiquitination of the Cytoplasmic Domain of Influenza A Virus M2 Protein Is Crucial for Production of Infectious Virus Particles. Journal of virology 92:e01972-01917.

33. García-Sastre, A., and P. Palese. 1995. The cytoplasmic tail of the neuraminidase protein of influenza A virus does not play an important role in the packaging of this protein into viral envelopes. Virus Res 37:37-47.

34. Jin, H., G. P. Leser, and R. A. Lamb. 1994. The influenza virus hemagglutinin cytoplasmic tail is not essential for virus assembly or infectivity. EMBO J 13:5504-5515. 
35. Kretzschmar, E., M. Bui, and J. K. Rose. 1996. Membrane Association of Influenza Virus Matrix Protein Does Not Require Specific Hydrophobic Domains or the Viral Glycoproteins. Virology 220:37-45.

36. Zhang, J., and R. A. Lamb. 1996. Characterization of the Membrane Association of the Influenza Virus Matrix Protein in Living Cells. Virology 225:255-266.

37. Chen, B. J., G. P. Leser, E. Morita, and R. A. Lamb. 2007. Influenza virus hemagglutinin and neuraminidase, but not the matrix protein, are required for assembly and budding of plasmid-derived virus-like particles. J. Virol. 81:7111-7123.

38. Dunsing, V., and S. Chiantia. 2018. A Fluorescence Fluctuation Spectroscopy Assay of Protein-Protein Interactions at Cell-Cell Contacts. JoVE:e58582.

39. Wagner, R., G. Gabriel, M. Schlesner, N. Alex, A. Herwig, O. Werner, and H.-D. Klenk. 2013. Protease activation mutants elicit protective immunity against highly pathogenic avian influenza viruses of subtype $\mathrm{H} 7$ in chickens and mice. Emerg Microbes Infect 2:e7-e7.

40. Wagner, R., A. Herwig, N. Azzouz, and H. D. Klenk. 2005. Acylation-Mediated Membrane Anchoring of Avian Influenza Virus Hemagglutinin Is Essential for Fusion Pore Formation and Virus Infectivity. Journal of Virology 79:6449.

41. Dunsing, V., M. Luckner, B. Zühlke, R. A. Petazzi, A. Herrmann, and S. Chiantia. 2018. Optimal fluorescent protein tags for quantifying protein oligomerization in living cells. Scientific reports 8:10634-10634.

42. Dunsing, V., A. Petrich, and S. Chiantia. 2020. Multi-color fluorescence fluctuation spectroscopy in living cells via spectral detection. elife 2021;10:e69687 DOI: 10.7554/eLife.69687

43. Wickham, H. 2016. ggplot2 - Elegant Graphics for Data Analysis. Springer International Publishing, New York.

44. Kassambara, A. 2020. ggpubr: 'ggplot2' Based Publication Ready Plots. R package version 0.4.0. https://CRAN.R-project.org/package=ggpubr.

45. Wilke, C. O. 2020. cowplot: Streamlined Plot Theme and Plot Annotations for 'ggplot2'. R package version 1.1.0. https://CRAN.R-project.org/package=cowplot.

46. Das, S. C., S. Watanabe, M. Hatta, T. Noda, G. Neumann, M. Ozawa, and Y. Kawaoka. 2012. The highly conserved arginine residues at positions 76 through 78 of influenza $A$ virus matrix protein $\mathrm{M} 1$ play an important role in viral replication by affecting the intracellular localization of M1. Journal of virology 86:1522-1530.

47. Thaa, B., A. Herrmann, and M. Veit. 2009. The polybasic region is not essential for membrane binding of the matrix protein M1 of influenza virus. Virology 383:150-155.

48. Chlanda, P., O. Schraidt, S. Kummer, J. Riches, H. Oberwinkler, S. Prinz, H. G. Kräusslich, and J. A. Briggs. 2015. Structural Analysis of the Roles of Influenza A Virus MembraneAssociated Proteins in Assembly and Morphology. J Virol 89:8957-8966.

49. Curthoys, N. M., M. J. Mlodzianoski, M. Parent, M. B. Butler, P. Raut, J. Wallace, J. Lilieholm, K. Mehmood, M. S. Maginnis, H. Waters, B. Busse, J. Zimmerberg, and S. T. Hess. 2019. Influenza Hemagglutinin Modulates Phosphatidylinositol 4,5Bisphosphate Membrane Clustering. Biophys J 116:893-909.

50. Digman, M. A., R. Dalal, A. F. Horwitz, and E. Gratton. 2008. Mapping the Number of Molecules and Brightness in the Laser Scanning Microscope. Biophysical Journal 94:2320-2332.

51. Petazzi, R. A., A. K. Aji, and S. Chiantia. 2020. Chapter One - Fluorescence microscopy methods for the study of protein oligomerization. In Progress in Molecular Biology and Translational Science. J. Giraldo, and F. Ciruela, editors. Academic Press. 1-41. 
52. Dunsing, V., M. Mayer, F. Liebsch, G. Multhaup, and S. Chiantia. 2017. Direct evidence of amyloid precursor-like protein 1 trans interactions in cell-cell adhesion platforms investigated via fluorescence fluctuation spectroscopy. Mol Biol Cell 28:3609-3620.

53. Scolari, S., S. Engel, N. Krebs, A. P. Plazzo, R. F. M. De Almeida, M. Prieto, M. Veit, and A. Herrmann. 2009. Lateral distribution of the transmembrane domain of influenza virus hemagglutinin revealed by time-resolved fluorescence imaging. The Journal of biological chemistry 284:15708-15716.

54. Varghese, J. N., W. G. Laver, and P. M. Colman. 1983. Structure of the influenza virus glycoprotein antigen neuraminidase at 2.9 ̊ resolution. Nature 303:35-40.

55. Georgieva, E. R., P. P. Borbat, H. D. Norman, and J. H. Freed. 2015. Mechanism of influenza A M2 transmembrane domain assembly in lipid membranes. Scientific reports 5:11757-11757.

56. Orekhova, A. S., P. S. Sverdlova, P. V. Spirin, O. G. Leonova, V. I. Popenko, V. S. Prasolov, and P. M. Rubtsov. 2011. [Novel bidirectional promoter from human genome]. Mol Biol (Mosk) 45:486-495.

57. Sengupta, P., A. Y. Seo, H. A. Pasolli, Y. E. Song, M. C. Johnson, and J. LippincottSchwartz. 2019. A lipid-based partitioning mechanism for selective incorporation of proteins into membranes of HIV particles. Nat Cell Biol 21:452-461.

58. Gao, X., and J. Zhang. 2008. Spatiotemporal analysis of differential Akt regulation in plasma membrane microdomains. Molecular biology of the cell 19:4366-4373.

59. Zacharias, D. A., J. D. Violin, A. C. Newton, and R. Y. Tsien. 2002. Partitioning of LipidModified Monomeric GFPs into Membrane Microdomains of Live Cells. Science 296:913.

60. Yeung, T., M. Terebiznik, L. Yu, J. Silvius, W. M. Abidi, M. Philips, T. Levine, A. Kapus, and S. Grinstein. 2006. Receptor Activation Alters Inner Surface Potential During Phagocytosis. Science 313:347.

61. Nayak, D. P., E. K. Hui, and S. Barman. 2004. Assembly and budding of influenza virus. Virus Res 106:147-165.

62. Zhao, H., and P. Lappalainen. 2012. A simple guide to biochemical approaches for analyzing protein-lipid interactions. Molecular biology of the cell 23:2823-2830.

63. Dawes, M. L., C. Soeller, and S. Scholpp. 2020. Studying molecular interactions in the intact organism: fluorescence correlation spectroscopy in the living zebrafish embryo. Histochem Cell Biol 154:507-519.

64. Langowski, J. 2008. Protein-protein interactions determined by fluorescence correlation spectroscopy. Methods Cell Biol 85:471-484.

65. Petazzi, R. A., A. A. Koikkarah, N. D. Tischler, and S. Chiantia. 2020. Detection of Envelope Glycoprotein Assembly from Old-World Hantaviruses in the Golgi Apparatus of Living Cells. J Virol.

66. Tzoneva, R., T. Stoyanova, A. Petrich, D. Popova, V. Uzunova, A. Momchilova, and S. Chiantia. 2020. Effect of Erufosine on Membrane Lipid Order in Breast Cancer Cell Models. Biomolecules 10.

67. Hoffmann, E., G. Neumann, Y. Kawaoka, G. Hobom, and R. G. Webster. 2000. A DNA transfection system for generation of influenza $A$ virus from eight plasmids. Proceedings of the National Academy of Sciences of the United States of America 97:6108-6113.

68. Rana, M. S., X. Wang, and A. Banerjee. 2018. An Improved Strategy for Fluorescent Tagging of Membrane Proteins for Overexpression and Purification in Mammalian Cells. Biochemistry 57:6741-6751. 
69. Snapp, E. 2005. Design and use of fluorescent fusion proteins in cell biology. Curr Protoc Cell Biol Chapter 21:21.24.21-21.24.13.

70. Cristian, L., J. D. Lear, and W. F. DeGrado. 2003. Use of thiol-disulfide equilibria to measure the energetics of assembly of transmembrane helices in phospholipid bilayers. Proc Natl Acad Sci U S A 100:14772-14777.

71. Barman, S., L. Adhikary, A. K. Chakrabarti, C. Bernas, Y. Kawaoka, and D. P. Nayak. 2004. Role of transmembrane domain and cytoplasmic tail amino acid sequences of influenza a virus neuraminidase in raft association and virus budding. Journal of virology 78:5258-5269.

72. Zhang, J., G. P. Leser, A. Pekosz, and R. A. Lamb. 2000. The cytoplasmic tails of the influenza virus spike glycoproteins are required for normal genome packaging. Virology 269:325-334.

73. Melkonian, K. A., A. G. Ostermeyer, J. Z. Chen, M. G. Roth, and D. A. Brown. 1999. Role of lipid modifications in targeting proteins to detergent-resistant membrane rafts. Many raft proteins are acylated, while few are prenylated. J Biol Chem 274:3910-3917.

74. Tatulian, S. A., and L. K. Tamm. 2000. Secondary structure, orientation, oligomerization, and lipid interactions of the transmembrane domain of influenza hemagglutinin. Biochemistry 39:496-507.

75. Iwatsuki-Horimoto, K., T. Horimoto, T. Noda, M. Kiso, J. Maeda, S. Watanabe, Y. Muramoto, K. Fujii, and Y. Kawaoka. 2006. The cytoplasmic tail of the influenza A virus M2 protein plays a role in viral assembly. J Virol 80:5233-5240.

76. McCown, M. F., and A. Pekosz. 2005. The influenza A virus M2 cytoplasmic tail is required for infectious virus production and efficient genome packaging. J Virol 79:3595-3605.

77. Zebedee, S. L., and R. A. Lamb. 1989. Growth restriction of influenza A virus by M2 protein antibody is genetically linked to the M1 protein. Proc Natl Acad Sci U S A 86:1061-1065.

78. Baer, A., and K. Kehn-Hall. 2014. Viral concentration determination through plaque assays: using traditional and novel overlay systems. Journal of visualized experiments : JoVE:e52065-e52065.

79. Petrášek, Z., and P. Schwille. 2008. Precise Measurement of Diffusion Coefficients using Scanning Fluorescence Correlation Spectroscopy. Biophysical Journal 94:14371448. 
80. Bacia, K., S. A. Kim, and P. Schwille. 2006. Fluorescence cross-correlation spectroscopy in living cells. Nature Methods 3:83-89.

81. Schneider, F., D. Waithe, M. P. Clausen, S. Galiani, T. Koller, G. Ozhan, C. Eggeling, and E. Sezgin. 2017. Diffusion of lipids and GPI-anchored proteins in actin-free plasma membrane vesicles measured by STED-FCS. Molecular biology of the cell 28:15071518.

82. Ries, J., and P. Schwille. 2006. Studying Slow Membrane Dynamics with Continuous Wave Scanning Fluorescence Correlation Spectroscopy. Biophysical Journal 91:19151924.

83. Ries, J., S. Chiantia, and P. Schwille. 2009. Accurate determination of membrane dynamics with line-scan FCS. Biophysical journal 96:1999-2008.

84. Linkert, M., C. T. Rueden, C. Allan, J.-M. Burel, W. Moore, A. Patterson, B. Loranger, J. Moore, C. Neves, D. MacDonald, A. Tarkowska, C. Sticco, E. Hill, M. Rossner, K. W. Eliceiri, and J. R. Swedlow. 2010. Metadata matters: access to image data in the real world. Journal of Cell Biology 189:777-782.

85. Trullo, A., V. Corti, E. Arza, V. R. Caiolfa, and M. Zamai. 2013. Application limits and data correction in number of molecules and brightness analysis. Microscopy Research and Technique 76:1135-1146.

86. Chen, Y., J. Johnson, P. Macdonald, B. Wu, and J. D. Mueller. 2010. Chapter 16 Observing Protein Interactions and Their Stoichiometry in Living Cells by Brightness Analysis of Fluorescence Fluctuation Experiments. In Methods in Enzymology. N. G. Walter, editor. Academic Press. 345-363. 